\title{
Mathematical theory and simulations of thermoporoelasticity
}

\author{
Cornelis J. van Duijn ${ }^{\mathrm{a}, 1}$, Andro Mikelić ${ }^{\mathrm{b}, 2, *}$, Thomas Wick ${ }^{\mathrm{c}}$ \\ ${ }^{a}$ Darcy Center, Eindhoven University of Technology, Department of Mechanical Engineering and \\ Utrecht University, Department of Earth Sciences, The Netherlands \\ ${ }^{b}$ Univ Lyon, Université Claude Bernard Lyon 1, CNRS UMR 5208, Institut Camille Jordan, 43 \\ blvd. du 11 novembre 1918, F-69622 Villeurbanne cedex, France \\ ${ }^{c}$ Leibniz Universität Hannover, Institut für Angewandte Mathematik, Welfengarten 1, 30167 \\ Hannover, Germany
}

\begin{abstract}
In this paper we study the equations of semi-linear thermoporoelasticity. Starting point is the dimensionless formulation in van Duijn et al [7] (C.J. van Duijn, A. Mikelić, M. F. Wheeler, T. Wick, Internat. J Engng Sci., Vol. 138, 2019), which was obtained by a formal two-scale expansion. Nonlinearities in the equations arise through the fluid viscosity and the thermal conductivity, both may depend on temperature, and through the coupling in the heat convection by the Darcy discharge in the energy equation. The coupled system of equations involves as unknowns the skeleton displacement, Darcy discharge, fluid pressure and temperature. We treat the system in its incremental (i.e. time-discrete) form. We prove existence by applying a fundamental theorem of Brézis on pseudo-monotone operators. Moreover we show that the free energy of the system acts as a Lyapunov functional. This yields global stability in the time-stepping process. Our theoretical results are substantiated with two-dimensional numerical tests using a monolithic formulation. Temporal discretization is based on the backward Euler scheme and finite elements are employed for the spatial discretization. The semi-linear discrete system is solved with Newton's method. In the proposed numerical examples, different source terms are employed and spatial mesh refinement studies show computational convergence.
\end{abstract}

Keywords: Thermoporoelasticity equations, heat convection by Darcy's velocity, free energy and stability, monolithic numerical scheme 


\section{Introduction}

In this paper we consider the equations for non-isothermal fluid flow through a deformable porous medium. Such equations arise, for example, in geothermal reservoir modeling when the withdrawal or injection of fluid affects the mechanical properties of the porous medium. They also arise in models for radioactive waste disposal in geological clay formations, or when modeling non-isothermal hydraulic fracturing in reservoirs, see e.g. [21, 17]. More details and related references are given in the work of McTigue [13], Lewis \& Schrefler [11, Section 10], and Rutquist et al [19].

This work is motivated by suggestions and collaborations with Professor M. F. Wheeler of the University of Texas at Austin. She pointed out the importance of thermoporoelasticity as well as the lack of a mathematical foundation of the equations involved. This work is a first step in that direction.

The emphasis here is on the mathematical analysis of the equations. Therefore we restrict the complexity of the underlying physical model and consider the case of a homogeneous and isotropic porous medium that is fully saturated with a single fluid.

We also consider the fluid density and the density of the skeleton material to be constant. However, we allow for a temperature dependence of the fluid viscosity and we impose a certain (quadratic) growth of the thermal conductivity with temperature. We return to this point at the end of this section, see Remark 1.

There are two main approaches that lead to the equations of thermoporoelasticity. One is based on mixture theory, see for instance Bredford and Drumheller [2], and uses the macroscopic mass balances of the fluid and solid grains as starting point. This approach is detailed in Coussy [5], Lewis \& Schrefler [11], and in the survey article of Rutquist et al [19]. It yields a coupled system of equations for the skeleton displacement, the fluid pressure and the temperature of the mixture.

The other is the multiscale approach, which uses as starting point the linear fluidskeleton interaction at the pore scale. This approach has a rigorous mathematical

\footnotetext{
${ }^{*}$ Corresponding author

Email addresses: C.J.v.Duijn@TUE.nl (Cornelis J. van Duijn ), mikelic@univ-lyon1.fr (Andro Mikelić ), thomas.wick@ifam.uni-hannover.de (Thomas Wick)

${ }^{1}$ CJvD acknowledges the support of the Darcy Center (Utrecht University - Eindhoven University of Technology) and of the Deutsche Forschungsgemeinschaft (DFG, German Research Foundation) for supporting this work by funding SFB 1313, Project Number 327154368.

${ }^{2}$ This work of A.M. benefited from the support of the project UNG=O UPGEO < ANR-19-CU05$032\rangle$ of the French National Research Agency (ANR) and from the LABEX MILYON (ANR-10LABX-0070) of Université de Lyon, within the program "Investissements d'Avenir" (ANR-11-IDEX0007) operated by the French National Research Agency (ANR).
} 
foundation in the isothermal case, see Mikelić \& Wheeler [15]. We also refer the reader to the monographs of Mei \& Vernescu [14] and Sanchez-Palencia [20] for derivations using two-scale expansions. In the non-isothermal case, the derivation of the macroscopic equations by means of a formal two-scale expansion was considered by Lee \& Mei $[9,10]$ and more recently by van Duijn et al [7]. The equations obtained in the latter paper form the basis of our analysis.

The equations on the pore scale involve physical quantities that appear in the description of the hydraulic, mechanical and thermal behavior. As shown in van Duijn et al [7], these quantities can be combined into a number of dimensionless parameters. This allows one to compare different physical effects. The result of the two-scale expansion is a (dimensionless) formulation in terms of pressure ( $p$, includes the hydraulic component ), skeleton displacement $\left(\mathbf{u}\right.$, a vector in $\left.\mathbb{R}^{m}(m=2,3)\right)$ and temperature $(\vartheta)$. It comprises the equations:

\section{Mass balance}

$$
\partial_{t} \zeta+a \operatorname{div} \mathbf{v}^{D}=Q,
$$

where

$$
\mathbf{v}^{D}=-\frac{k}{\eta(\vartheta)} \nabla p
$$

and

$$
\zeta=\frac{p-K_{S} \vartheta}{M}+b \operatorname{div} \mathbf{u}
$$

Momentum balance

$$
-\operatorname{div} \sigma=\mathbf{F},
$$

where

$$
\sigma=\mathcal{G} e(\mathbf{u})-\left(b p+(1-b) K_{S} \vartheta\right) \mathbb{I},
$$

and

$$
\mathcal{G} e(\mathbf{u})=2 \mu e(\mathbf{u})+\lambda \operatorname{div} \mathbf{u} \mathbb{I} .
$$

Energy balance 


$$
\frac{\partial \vartheta}{\partial t}+K_{T} \frac{\partial}{\partial t}(-\zeta+\operatorname{div} \mathbf{u})+C_{f} \mathbf{v}^{D} \cdot \nabla \vartheta-\operatorname{div}(\beta(\vartheta) \nabla \vartheta)=H
$$

In this expression $\zeta$ is the incremental fluid content, $e(\mathbf{u})=\frac{1}{2}\left(\nabla \mathbf{u}+\nabla \mathbf{u}^{\tau}\right)$ the linearized strain tensor, $\mathbf{v}^{D}$ the Darcy specific discharge, $\eta=\eta(\vartheta)$ the temperature dependent viscosity and $\beta(\vartheta)$ the temperature dependent thermal diffusivity. Further $Q$ and $H$ are source terms for, respectively, fluid volume and heat, and $\mathbf{F}$ is a given body force. All other quantities are dimensionless parameters ${ }^{3}$. They are listed in Table 1, where also their order of magnitude is given for typical rock and clay type materials. This table is essentially taken from van Duijn et al [7].

\begin{tabular}{|c|c|c|c|}
\hline Symbol & Quantity & Rock value & Clay value \\
\hline$a$ & $\begin{array}{l}\text { macroscopic length/ characteristic } \\
\text { displacement or transport time/ } \\
\text { consolidation time }\end{array}$ & $10^{3}$ & $10^{2}$ \\
\hline$b$ & Biot's coefficient & $O(1)$ & $O(1)$ \\
\hline $1 / M$ & compressibility coefficient & $O\left(10^{-1}\right)$ & $O\left(10^{-1}\right)$ \\
\hline$k$ & permeability & $O\left(10^{-4}\right)$ & $O\left(10^{-4}\right)$ \\
\hline$\beta_{0}$ & thermal diffusivity & $O(1)$ & $O(1)$ \\
\hline$K_{S}$ & solid expansion coefficient & $O\left(10^{-2}\right)$ & $O\left(10^{-3}\right)$ \\
\hline$K_{T}$ & thermoelastic coupling parameter & $O\left(10^{-4}\right)$ & $O\left(10^{-7}\right)$ \\
\hline$\mu, \lambda$ & Lamé's coefficients & $O(1)$ & $O(1)$ \\
\hline$C_{f}$ & fractional heat capacity & $O(1)$ & $O(1)$ \\
\hline
\end{tabular}

Table 1: Dimensionless parameters and order of magnitude

We consider equations (1)-(7) in a bounded domain $\Omega \subset \mathbb{R}^{m}(m=2,3)$, whose boundary $\partial \Omega$ is $C^{1}$, and we take the time $t \in(0, T]$, where $T>0$ may be arbitrarily chosen. As initial-boundary conditions we have

$$
\operatorname{div} \mathbf{u}=\mathcal{E}_{0}, \vartheta=\vartheta_{\text {in }} \quad \text { and } \quad p=p_{\text {in }} \quad \text { in } \Omega \text { as } t=0
$$

and

$$
\mathbf{u}=0, \quad \mathbf{v}^{D} \cdot \mathbf{v}=q^{d} \text { and } \quad \beta(\vartheta) \nabla \vartheta \cdot v=0 \quad \text { at } \quad \partial \Omega \quad \text { for } \quad 0<t \leq T .
$$

\footnotetext{
${ }^{3}$ The coefficient $(1-b) K_{S}$ is frequently taken as an independent parameter, not directly linked to $K_{S}$ or $b$ (see e.g. [5]). Our results remain valid if $(1-b) K_{S}$ is replaced in the momentum and energy equations by some other parameter.
} 
Hence along the boundary of the domain, the system is mechanically fixed, isolated for heat flow, but with prescribed fluid flow. We could have considered more general boundary conditions. The choice in (9) was made for simplicity.

In equation ( 7$)$ the heat transport has the form $\mathbf{v}^{D} \cdot \nabla \vartheta$, i.e. with Darcy discharge multiplying the gradient of the temperature. This is a direct consequence of the ALE (Arbitrary Lagrangian-Euler) formulation used in van Duijn et al [7]. This term also arises when applying the full Eulerian formulation in the energy equation at the pore scale, as was shown in Lee \& Mei [9, 10]. In Lewis \& Schrefler [11] or Néron \& Dureisseix [16] it was derived from the mixture theory of Bredford \& Drumheller [2].

Alternatively, one uses in the macroscopic description a direct formulation in terms of the heat flux. This results in the convective transport term

$$
\operatorname{div}\left(\vartheta \mathbf{v}^{D}\right)
$$

Clearly, when $\operatorname{div} \mathbf{v}^{D}=0$, both formulations coincide.

In this paper we mainly focus on (7). However, we do show that if (10) is used, the analysis goes along the same lines.

An important role in the analysis is played by the free energy associated with system (1)-(7). As in van Duijn et al [7] it is given by

$$
\mathcal{L}=\frac{1}{2} \mathcal{G} e: e+\frac{\left(p-K_{S} \vartheta\right)^{2}}{2 M}+\frac{K_{S} \vartheta^{2}}{2 K_{T}}-\mathbf{F} \cdot \mathbf{u}
$$

or, in terms of the incremental fluid content $\zeta$,

$$
\mathcal{L}=\frac{1}{2} \mathcal{G} e: e+\frac{(\zeta-b e: \mathbb{I})^{2}}{2 M}+\frac{K_{S} \vartheta^{2}}{2 K_{T}}-\mathbf{F} \cdot \mathbf{u}
$$

This expression reduces to the free energy in Biot [3] when $\vartheta=0$, i.e. the isothermal case. It also has qualitatively the same form as the reduced potential in Coussy et al [6]. Note that from (12)

$$
\frac{\partial \mathcal{L}}{\partial e}=\sigma+K_{S} \vartheta \mathbb{I} \quad \text { and } \quad \frac{\partial \mathcal{L}}{\partial \zeta}=p-K_{S} \vartheta .
$$

In this paper we treat the time discrete version of system (1)-(7), i.e. we replace time derivative in (1)-(7) by backwards finite differences.

In Section 2 we prove existence for the corresponding incremental problem and we show that

$$
\int_{\Omega} \mathcal{L} d x
$$


acts as a Lyapunov functional. This ensures global stability for the time discrete problem. Section 3 gives equivalent results for the case where the heat convection is given by expression (10). In Section 4 we demonstrate the effect of the thermomechanical coupling by means of several numerical examples. Conclusions are given in Section 5.

Remark 1. The existence proof of the incremental problem borrows ideas from van Duijn 85 Mikelic [8]. Key point is to formulate the time discrete form of (1)-(7) in an abstract framework and to show that the resulting operator is pseudo-monotone and coercive. This approach was taken from the monograph of Rubiček [18], who in turn uses the theory of pseudo-monotone operators of Brézis [12]. To prove coercivity of the operator and to show that (14) is a Lyapunov functional, we need to assume that the thermal diffusivity $\beta(\vartheta)$ satisfies a growth condition. This is required to balance the effect of the thermal transport in the weak formulation. With respect to the viscosity and diffusivity we assume

A1 $\eta \in C(\mathbb{R})$ and there exist constants $0<\eta_{\min }<\eta_{\max }$ such that $\eta_{\min } \leq \eta(\vartheta) \leq \eta_{\max }$ for all $\vartheta \in \mathbb{R}$;

A2 $\beta \in C(\mathbb{R})$ and there exist constants $0<C_{\min }<C_{\max }$ and $\beta_{0}>0$ such that

$$
\beta_{0}+C_{\min } \vartheta^{2} \leq \beta(\vartheta) \leq \beta_{0}+C_{\text {max }} \vartheta^{2} \quad \text { for all } \vartheta \in \mathbb{R}
$$

Here

$$
C_{\text {min }} \geq \frac{1}{a} \frac{K_{S}}{K_{T}} \frac{C_{f}^{2} k}{\eta_{\min }} .
$$

According to Table 1, we have

$$
C_{\min } \geq \begin{cases}10^{-5}, & \text { rock values } \\ 10^{-2}, & \text { clay values }\end{cases}
$$

Since $\vartheta=O\left(\frac{\Delta T}{T_{0}}\right)$, where $\Delta T$ is a characteristic temperature variation and $T_{0}$ a reference temperature, we have in practical terms $\vartheta=O\left(10^{-1}\right)$ for which the lower bound is nearly constant. Only for very large (unrealistic) values of $\vartheta$ the growth of the lower bound has significance.

With respect to the other data we have

A3 $Q, H \in C\left([0, T] ; L^{2}(\Omega)\right), \mathbf{F} \in C\left([0, T] ; L^{2}(\Omega)\right)^{m}$ and $q^{d} \in C\left([0, T] ; L^{2}(\partial \Omega)\right)$; $\mathcal{E}_{0} \in L^{2}(\Omega)$. 
Remark 2. (Compatibility). As shown in this paper, conditions (8) and (9) suffice for the existence of the incremental problem. However, in the numerical solutions or when considering the continuous time case, e.g. see [8], one needs in addition the compatibility condition

$$
\int_{\Omega} \mathcal{E}_{0} d x=0
$$

\section{Existence and stability for time discrete problem}

Our goal is to develop a mathematical theory for semi-linear thermoporoelasticity, giving a stable discretization in a time. Since most of the thermodynamic modeling corresponds to the time discrete quasi-static setting (see for instance [5]), we limit our consideration to the incremental formulation. Correct modeling of the continuous time case would require taking into the account other non-stationary phenomena. This is outside the scope of this paper.

Following the classical time discretization of parabolic PDEs, we choose a small discretization step $\tau>0$ and an integer $N$, such that $N=T / \tau$. At each discrete time $t_{j}=j \tau, j=0,1, \ldots, N$, we set

$$
\mathbf{u}^{j}(x)=\mathbf{u}\left(x, t_{j}\right), \vartheta^{j}(x)=\vartheta\left(x, t_{j}\right), p^{j}(x)=p\left(x, t_{j}\right), \quad x \in \Omega,
$$

for the unknown functions and

$\mathbf{F}^{j}(x)=\mathbf{F}\left(x, t_{j}\right), Q^{j}(x)=Q\left(x, t_{j}\right), H^{j}(x)=H\left(x, t_{j}\right), x \in \Omega ; q^{d, j}(x)=q^{d}\left(x, t_{j}\right), x \in \partial \Omega$ for given body forces, source/sink terms and boundary terms.

For convenience we rewrite (7) as

$\frac{\partial}{\partial t}\left(a_{0} \vartheta-\frac{K_{S}}{M} p+(1-b) K_{S} \operatorname{divu}\right)+\frac{K_{S} C_{f}}{K_{T}} \mathbf{v}^{D} \cdot \nabla \vartheta-\frac{K_{S}}{K_{T}} \operatorname{div}(\beta(\vartheta) \nabla \vartheta)=\frac{K_{S}}{K_{T}} H=: H$,

where $a_{0}=K_{S} / K_{T}+K_{S}^{2} / M$.

Then we replace (2)-(6), (18) by its time discrete form, for $j=1, \ldots, N$,

$$
\begin{gathered}
-\operatorname{div}\left(2 \mu e_{x}\left(\mathbf{u}^{j}\right)+\lambda \operatorname{div} \mathbf{u}^{j} I-b p^{j} \mathbb{I}-(1-b) K_{S} \vartheta^{j} \mathbb{I}\right)=\mathbf{F}^{j}, \\
\partial_{\tau}\left(\frac{p^{j}-K_{S} \vartheta^{j}}{M}+b \operatorname{div} \mathbf{u}^{j}\right)-a \operatorname{div}\left(\frac{k}{\eta\left(\vartheta^{j}\right)} \nabla p^{j}\right)=Q^{j}, \\
\partial_{\tau}\left(a_{0} \vartheta^{j}-\frac{K_{S}}{M} p^{j}+(1-b) K_{S} \operatorname{div} \mathbf{u}^{j}\right)+\frac{K_{S} C_{f}}{K_{T}} \mathbf{v}^{D, j} \cdot \nabla \vartheta^{j}- \\
\frac{K_{S}}{K_{T}} \operatorname{div}\left(\beta\left(\vartheta^{j}\right) \nabla \vartheta^{j}\right)=H^{j},
\end{gathered}
$$


with

$$
\mathbf{v}^{D, j}=-\frac{k}{\eta\left(\vartheta^{j}\right)} \nabla p^{j}
$$

Here $\partial_{\tau}$ denotes the backward time difference, i.e. $\partial_{\tau} \Phi:=\left(\Phi^{j}-\Phi^{j-1}\right) / \tau$.

Initially, for $j=0$, we have

$$
\operatorname{div} \mathbf{u}^{0}=\mathcal{E}_{0}, \vartheta^{0}=\vartheta_{\text {in }} \text { and } p^{0}=p_{\text {in }},
$$

and at the boundary $\partial \Omega$ we have

$$
\mathbf{u}^{j}=0, \quad \mathbf{v}^{D, j} \cdot \boldsymbol{v}=q^{d, j} \quad \text { and } \quad \beta(\vartheta) \nabla \vartheta \cdot v=0 .
$$

\subsection{Existence of a solution to the incremental problem}

The variational (or virtual work) formulation of equations (19)-(22), initial conditions (23) and boundary conditions (24), reads:

Problem (IP) (incremental problem): $\quad$ Let $W:=H_{0}^{1}(\Omega)^{m} \times H^{1}(\Omega)^{2}$. Then for given $(\mathbf{U}, \pi, \Theta) \in W$, find $(\mathbf{u}, p, \vartheta) \in W, \sqrt{\beta(\vartheta)} \nabla \vartheta \in L^{2}(\Omega)^{m}$, such that

$$
\begin{gathered}
\int_{\Omega} 2 \mu e(\mathbf{u}): e(\xi) d x-\int_{\Omega}\left(b p+(1-b) K_{S} \vartheta-\lambda \operatorname{divu}\right) \operatorname{div} \xi d x d z=\int_{\Omega} \mathbf{F} \xi d x \\
\forall \xi \in H_{0}^{1}(\Omega)^{m} \cdot \\
\int_{\Omega} \partial_{\tau}\left(\frac{p-K_{S} \vartheta}{M}+b \operatorname{div} \mathbf{u}\right) \psi d x+a \int_{\Omega} \frac{k}{\eta(\vartheta)} \nabla p \cdot \nabla \psi d x= \\
\int_{\Omega} Q \psi d x-\int_{\partial \Omega} q^{d} \psi d S, \quad \forall \psi \in H^{1}(\Omega) \\
\int_{\Omega} \partial_{\tau}\left(a_{0} \vartheta-\frac{K_{S}}{M} p+(1-b) K_{S} \operatorname{div} \mathbf{u}\right) \theta d x+\frac{K_{S} C_{f}}{K_{T}} \int_{\Omega} \mathbf{v}^{D} \cdot \nabla \vartheta \theta d x+ \\
\frac{K_{S}}{K_{T}} \int_{\Omega} \beta(\vartheta) \nabla \vartheta \cdot \nabla \theta d x=\int_{\Omega} H \theta d x, \quad \forall \theta \in W^{1, r}(\Omega), r>m
\end{gathered}
$$

Remark 3. (i) Note that the "initial" conditions $(\mathbf{U}, \pi, \Theta)$ are hidden in the backward time differences:

$$
\partial_{\tau} \mathbf{u}=\frac{\mathbf{u}-\mathbf{U}}{\tau}, \quad \partial_{\tau} p=\frac{p-\pi}{\tau} \quad \text { and } \quad \partial_{\tau} \vartheta=\frac{\vartheta-\Theta}{\tau} .
$$

(ii) Only for numerical purposes one needs $\mathbf{U}$ as initial condition. In (IP) only div $\mathbf{U}$ is needed as initial condition. 
(iii) Concerning the notation in problem (IP): the data, i.e. $Q, H, \mathbf{F}$ and $q^{d}$ are taken at times that correspond to the time of the discrete solution $(\mathbf{u}, p, \vartheta)$. Superscripts are therefore omitted.

Problem (IP) is semi-linear through the nonlinearities $\eta(\vartheta)$ and $\beta(\vartheta)$ and through the heat convection due to the Darcy discharge

$$
\int_{\Omega}\left(\mathbf{v}^{D} \cdot \nabla \vartheta\right) \theta d x .
$$

Using the weak topology of $W$, problems arise with the continuity of expression (28). In fact, for $\theta \in H^{1}(\Omega)(28)$ is not defined. No a priori information on the regularity of div $\mathbf{v}^{D}$ is available and no maximum principle for $\vartheta$ is to be expected.

To remedy this difficulty in the incremental setting, we follow the approach from [8] and introduce a Friedrichs mollifier $\mathcal{K}_{\varepsilon}$, where $\varepsilon$ is a small positive parameter (see e.g. Rubiček [18, page 203]). The Darcy velocity $\mathbf{v}^{D}$ in the nonlinear heat convection term is replaced by the convolution $\mathbf{v}^{D} \star \mathcal{K}_{\varepsilon}$. Furthermore, in the same term and in the heat conduction term, $\vartheta$ is replaced by the cut-off

$$
R_{\varepsilon}(\vartheta)= \begin{cases}1 / \varepsilon, & \text { for } \vartheta>1 / \varepsilon \\ \vartheta, & \text { for }|\vartheta| \leq 1 / \varepsilon \\ -1 / \varepsilon, & \text { for } \vartheta<-1 / \varepsilon\end{cases}
$$

These changes allow us to use the theory of pseudo-monotone operators to study the following regularized incremental problem:

$$
\begin{gathered}
\text { Problem }(\mathbf{I P})_{\varepsilon}: \quad \text { Given }(\mathbf{U}, \pi, \Theta) \in W, \text { find }\left(\mathbf{u}^{\varepsilon}, p^{\varepsilon}, \vartheta^{\varepsilon}\right) \in W \text { such that } \\
\int_{\Omega} 2 \mu e\left(\mathbf{u}^{\varepsilon}\right): e(\xi) d x-\int_{\Omega}\left(b p^{\varepsilon}+(1-b) K_{S} \vartheta^{\varepsilon}-\lambda \operatorname{div} \mathbf{u}^{\varepsilon}\right) \operatorname{div} \xi d x=\int_{\Omega} \mathbf{F} \xi d x \\
\forall \xi \in H_{0}^{1}(\Omega)^{3} . \\
\int_{\Omega} \partial_{\tau}\left(\frac{p^{\varepsilon}-K_{S} \vartheta^{\varepsilon}}{M}+b \operatorname{div} \mathbf{u}^{\varepsilon}\right) \psi d x+a \int_{\Omega} \frac{k}{\eta\left(\vartheta^{\varepsilon}\right)} \nabla p^{\varepsilon} \cdot \nabla \psi d x= \\
\int_{\Omega} Q \psi d x-\int_{\partial \Omega} q^{d} \psi d S, \quad \forall \psi \in H^{1}(\Omega) ; \\
\int_{\Omega} \partial_{\tau}\left(a_{0} \vartheta^{\varepsilon}-\frac{K_{S}}{M} p^{\varepsilon}+(1-b) K_{S} \operatorname{div}^{\varepsilon}\right) \theta d x+\frac{K_{S} C_{f}}{K_{T}} \int_{\Omega} \mathcal{K}_{\varepsilon} \star \mathbf{v}^{D, \varepsilon} \cdot \nabla R_{\varepsilon}\left(\vartheta^{\varepsilon}\right) \theta d x+ \\
\frac{K_{S}}{K_{T}} \int_{\Omega} \beta\left(R_{\varepsilon}\left(\vartheta^{\varepsilon}\right)\right) \nabla \vartheta^{\varepsilon} \cdot \nabla \theta d x=\int_{\Omega} H \theta d x, \quad \forall \theta \in H^{1}(\Omega) .
\end{gathered}
$$


Remark 4. Note that in problem $(\mathbf{I P})_{\varepsilon}$, the temperature test function $\theta \in H^{1}(\Omega)$. This is allowed because of the presence of the cutoff $R_{\varepsilon}(\vartheta)$ in equation (31) and the boundedness of $\mathcal{K}_{\varepsilon} \star \mathbf{v}^{D, \varepsilon}$. Later, this allows us to test (31) with the solution $\vartheta^{\varepsilon} \in$ $H^{1}(\Omega)$.

Proposition 5. Let $\varepsilon>0$ be a small positive constant and let assumptions $A 1-3$ be satisfied. Then Problem $(\mathbf{I P})_{\varepsilon}$ admits at least one solution $\left(\mathbf{u}^{\varepsilon}, p^{\varepsilon}, \vartheta^{\varepsilon}\right) \in W$.

Proof. Following Rubiček [18] and van Duijn \& Mikelić [8], we introduce a nonlinear operator $\mathcal{A}$, defined on $W$ and with values in its dual $W^{\prime}$. It results from adding $\frac{1}{\tau} \cdot(29),(30)$ and $(31)$, which we write as, with $(\mathbf{u}, p, \vartheta) \in W$,

$$
\mathcal{A}(\mathbf{u}, p, \vartheta)=\mathbf{b}
$$

where

$$
\begin{gathered}
\langle\mathcal{A}(\mathbf{u}, p, \vartheta),(\xi, \psi, \theta)\rangle:=\frac{1}{\tau} \int_{\Omega} 2 \mu e(\mathbf{u}): e(\xi) d x-\frac{1}{\tau} \int_{\Omega}\left(\left(b p+(1-b) K_{S} \vartheta(1-b)\right.\right. \\
\left.-\lambda \operatorname{divu}) \operatorname{div} \xi+\left(\frac{p-K_{S} \vartheta}{M}+b \operatorname{div} \mathbf{u}\right) \psi+\left(a_{0} \vartheta-\frac{K_{S}}{M} p+(1-b) K_{S} \operatorname{divu}\right) \theta\right) d x \\
+a \int_{\Omega} \frac{k}{\eta(\vartheta)} \nabla p \cdot \nabla \psi d x+\frac{K_{S} C_{f}}{K_{T}} \int_{\Omega} \mathcal{K}_{\varepsilon} \star \mathbf{v}^{D} \cdot \nabla R_{\varepsilon}(\vartheta) \theta d x+ \\
\frac{K_{S}}{K_{T}} \int_{\Omega} \beta\left(R_{\varepsilon}(\vartheta)\right) \nabla \vartheta \cdot \nabla \theta d x, \quad \forall(\xi, \psi, \theta) \in W
\end{gathered}
$$

with $\mathbf{v}^{D}=-k \nabla p / \eta(\vartheta)$, and

$$
\begin{gathered}
\langle\mathbf{b},(\xi, \psi, \theta)\rangle:=\frac{1}{\tau} \int_{\Omega}\left(\mathbf{F} \cdot \xi+\left(\frac{\pi-K_{S} \Theta}{M}+b \operatorname{div} \mathbf{U}\right) \psi+\left(a_{0} \Theta-\frac{K_{S}}{M} \pi+\right.\right. \\
\left.\left.(1-b) K_{S} \operatorname{div} \mathbf{U}\right) \theta\right) d x+\int_{\Omega} Q \psi d x-\int_{\partial \Omega} q^{d} \psi d S+\int_{\Omega} H \theta d x, \quad \forall(\xi, \psi, \theta) \in W .
\end{gathered}
$$

Obviously $\mathbf{b} \in W^{\prime}$.

The idea is to show that $\mathcal{A}$ is a perturbed monotone operator: i.e. $\mathcal{A}$ is monotone in its principal part containing derivatives of $\mathbf{u}, p$ and $\vartheta$. To be precise, we show that $\mathcal{A}$ is pseudomonotone and coercive. This allows us to apply Brézis' theorem to (32) (see [18, chapter 2]) to conclude existence for problem $(\mathbf{I P})_{\varepsilon}$. 
For the comfort of the reader we recall that an operator $\mathcal{A}: W \rightarrow W^{\prime}$ is pseudomonotone if and only if $\mathcal{A}$ is bounded and

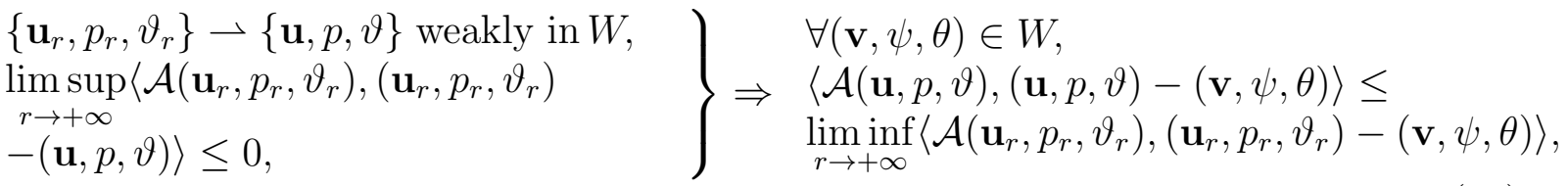

The boundedness of $\mathcal{A}$ is immediate. It is enough to show that (35) holds for the thermal convection operator

$$
\left\langle\mathcal{A}^{T}(p, \vartheta), \theta\right\rangle:=\int_{\Omega} \mathcal{K}_{\varepsilon} \star \mathbf{v}^{D} \cdot \nabla R_{\varepsilon}(\vartheta) \theta d x .
$$

For the other terms in $\mathcal{A}$ weak continuity and lower weak continuity are clear.

The weak convergence in $W$ implies weak $L^{2}$-convergence of $\mathbf{v}_{r}^{D}=k \nabla p_{r} / \eta\left(\vartheta_{r}\right)$. Using the regularization properties of the convolution, we then have as $r \rightarrow \infty$

$$
\mathcal{K}_{\varepsilon} \star \mathbf{v}_{r}^{D} \rightarrow \mathcal{K}_{\varepsilon} \star \mathbf{v}^{D}, \quad \text { uniformly in } \bar{\Omega} .
$$

Hence as $r \rightarrow \infty$, we have

$$
\begin{gathered}
\left\langle\mathcal{A}^{T}\left(p_{r}, \vartheta_{r}\right), \theta\right\rangle=\int_{\Omega} \mathcal{K}_{\varepsilon} \star \mathbf{v}_{r}^{D} \cdot \nabla R_{\varepsilon}\left(\vartheta_{r}\right) \theta d x \rightarrow \int_{\Omega} \mathcal{K}_{\varepsilon} \star \mathbf{v}^{D} \cdot \nabla R_{\varepsilon}(\vartheta) \theta d x ; \\
\text { and } \\
\left\langle\mathcal{A}^{T}\left(p_{r}, \vartheta_{r}\right), \theta\right\rangle=\int_{\Omega} \mathcal{K}_{\varepsilon} \star \mathbf{v}_{r}^{D} \cdot \underbrace{\nabla R_{\varepsilon}\left(\vartheta_{r}\right)}_{\text {weak } L^{2} \text { conv }} \underbrace{\vartheta_{r}}_{\text {strong } L^{2} \text { conv. }} d x \rightarrow \int_{\Omega} \mathcal{K}_{\varepsilon} \star \mathbf{v}^{D} \cdot \nabla R_{\varepsilon}(\vartheta) \vartheta d x .
\end{gathered}
$$

This establishes pseudo monotonicity for $\mathcal{A}^{T}$ and hence for $\mathcal{A}$.

It remains to prove coercivity of the operator $\mathcal{A}$.

Taking $\xi=\mathbf{u}, \psi=p$ and $\theta=\vartheta$ in (33) yields

$$
\begin{gathered}
\langle\mathcal{A}(\mathbf{u}, p, \vartheta),(\mathbf{u}, p, \vartheta)\rangle:=\frac{2 \mu}{\tau} \int_{\Omega}|e(\mathbf{u})|^{2} d x+\frac{1}{\tau} \int_{\Omega}\left(\lambda|\operatorname{div} \mathbf{u}|^{2}+\frac{\left(p-K_{S} \vartheta\right)^{2}}{M}+\frac{K_{S} \vartheta^{2}}{K_{T}}\right) d x \\
+a \int_{\Omega} \frac{k}{\eta(\vartheta)}|\nabla p|^{2} d x+\frac{K_{S} C_{f}}{K_{T}} \int_{\Omega} \mathcal{K}_{\varepsilon} \star \mathbf{v}^{D} \cdot \nabla R_{\varepsilon}(\vartheta) \vartheta d x+\frac{K_{S}}{K_{T}} \int_{\Omega} \beta\left(R_{\varepsilon}(\vartheta)\right)|\nabla \vartheta|^{2} d x,
\end{gathered}
$$

with $\mathbf{v}^{D}=-k \nabla p / \eta(\vartheta)$. 
By Young's convolution inequality we have

$$
\left\|\mathcal{K}_{\varepsilon} \star \mathbf{v}^{D}\right\|_{L^{2}(\Omega)} \leq\left\|\mathbf{v}^{D}\right\|_{L^{2}(\Omega)} .
$$

Hence the thermal transport term is estimated by, for any $\delta>0$ and with $\chi_{[-1 / \varepsilon, 1 / \varepsilon]}(\cdot)$ denoting the characteristic function of the interval $[-1 / \varepsilon, 1 / \varepsilon]$,

$$
\left|\int_{\Omega} \mathcal{K}_{\varepsilon} \star \mathbf{v}^{D} \cdot \nabla R_{\varepsilon}(\vartheta) \vartheta d x\right| \leq \frac{\delta}{2}\left\|\mathbf{v}^{D}\right\|_{L^{2}(\Omega)}^{2}+\frac{1}{2 \delta}\left\|\chi_{[-1 / \varepsilon, 1 / \varepsilon]}(\vartheta) \vartheta \nabla \vartheta\right\|_{L^{2}(\Omega)}^{2}
$$

Choosing $\delta=\frac{a K_{T} \eta_{\min }}{k K_{S} C_{f}}$ and using $\chi_{[-1 / \varepsilon, 1 / \varepsilon]}(\vartheta) \vartheta^{2} \leq R_{\varepsilon}^{2}(\vartheta)$, this gives

$$
\begin{gathered}
\frac{K_{S} C_{f}}{K_{T}} \int_{\Omega} \mathcal{K}_{\varepsilon} \star \mathbf{v}^{D} \cdot \nabla R_{\varepsilon}(\vartheta) \vartheta d x \geq-\frac{a \eta_{\min }}{2 k}\left\|\mathbf{v}^{D}\right\|_{L^{2}(\Omega)}^{2}- \\
\frac{k}{2 a \eta_{\min }}\left(\frac{K_{S} C_{f}}{K_{T}}\right)^{2}\left\|R_{\varepsilon}(\vartheta) \nabla \vartheta\right\|_{L^{2}(\Omega)}^{2} .
\end{gathered}
$$

Then we estimate in (36), using assumptions A1 and A2,

$$
\begin{gathered}
a \int_{\Omega} \frac{k}{\eta(\vartheta)}|\nabla p|^{2} d x+\frac{K_{S} C_{f}}{K_{T}} \int_{\Omega} \mathcal{K}_{\varepsilon} \star \mathbf{v}^{D} \cdot \nabla R_{\varepsilon}(\vartheta) \vartheta d x+\frac{K_{S}}{K_{T}} \int_{\Omega} \beta\left(R_{\varepsilon}(\vartheta)\right)|\nabla \vartheta|^{2} d x \\
\geq \frac{a \eta_{\text {min }}}{2 k}\left\|\mathbf{v}^{D}\right\|_{L^{2}(\Omega)}^{2}+\frac{K_{S}}{K_{T}} \int_{\Omega}\left(\beta\left(R_{\varepsilon}(\vartheta)\right)-\frac{1}{2 a} \frac{K_{S}}{K_{T}} \frac{C_{f}^{2} k}{\eta_{\min }} R_{\varepsilon}^{2}(\vartheta)\right)|\nabla \vartheta|^{2} d x \\
\geq \frac{a k}{2} \frac{\eta_{\text {min }}}{\eta_{\text {max }}^{2}}\|\nabla p\|_{L^{2}(\Omega)^{m}}^{2}+\frac{K_{S}}{K_{T}} \beta_{0}\|\nabla \vartheta\|_{L^{2}(\Omega)^{m}}^{2}+\frac{k}{2 a \eta_{\min }}\left(\frac{K_{S} C_{f}}{K_{T}}\right)^{2}\left\|R_{\varepsilon}(\vartheta) \nabla \vartheta\right\|_{L^{2}(\Omega)^{m}}^{2} .
\end{gathered}
$$

This shows the coercivity of $\mathcal{A}$ and completes the proof.

Corollary 6. We have

$$
\operatorname{div} \mathbf{v}^{D, \varepsilon}=-\operatorname{div}\left(\frac{k}{\eta\left(\vartheta^{\varepsilon}\right)} \nabla p^{\varepsilon}\right) \in L^{2}(\Omega)^{m} .
$$

Proof. Testing (32) with $\xi=0, \theta=0$ and arbitrary $\psi \in H^{1}(\Omega)$ gives the weak form of (20), (24). Then the result is immediate.

Proposition 7. Let $A 1-A 3$ be satisfied. Then Problem (IP) admits at least one solution $(\mathbf{u}, p, \vartheta) \in W$, such that $\sqrt{\beta(\vartheta)} \nabla \vartheta \in L^{2}(\Omega)^{3}$. 
Proof. Dropping the last term in (37) yields a coercivity estimate that is independent of $\varepsilon$. From this we deduce that there exists a constant $C>0$, not depending on $\varepsilon$, for which the coercivity estimate from the proof of Proposition 5 is independent of the regularization parameter $\varepsilon$. Hence we have

$$
\left\|\mathbf{u}^{\varepsilon}\right\|_{H_{0}^{1}(\Omega)^{3}}+\left\|p^{\varepsilon}\right\|_{H^{1}(\Omega)}+\left\|\vartheta^{\varepsilon}\right\|_{H^{1}(\Omega)} \leq C
$$

and

$$
\left\|\operatorname{div} \mathbf{v}^{D, \varepsilon}\right\|_{L^{2}(\Omega)} \leq C
$$

From these estimates, using weak compactness, we conclude that a subsequence $\left\{\left(\mathbf{u}^{\varepsilon}, p^{\varepsilon}, \vartheta^{\varepsilon}\right)\right\}$, denoted by the same subscript, and a triple $(\mathbf{u}, p, \vartheta) \in W$ exists such that

$$
\begin{gathered}
\mathbf{u}^{\varepsilon} \rightarrow \mathbf{u} \quad \text { weakly in } H_{0}^{1}(\Omega)^{3}, \\
\operatorname{div} \mathbf{u}^{\varepsilon} \rightarrow \operatorname{div} \mathbf{u} \text { weakly in } L^{2}(\Omega), \\
p^{\varepsilon} \rightarrow p \quad \text { weakly in } H^{1}(\Omega), \\
p^{\varepsilon} \rightarrow p \quad \text { strongly in } L^{2}(\Omega) \text { and }(\text { a.e }) \text { on } \Omega, \\
\vartheta^{\varepsilon} \rightarrow \vartheta \quad \text { weakly in } H^{1}(\Omega), \\
\vartheta^{\varepsilon} \rightarrow \vartheta \quad \text { strongly in } L^{2}(\Omega) \text { and (a.e) on } \Omega, \\
\mathbf{v}^{D, \varepsilon}=-\frac{k}{\eta\left(\vartheta^{\varepsilon}\right)} \nabla p^{\varepsilon} \rightarrow \mathbf{v}^{D}=-\frac{k}{\eta(\vartheta)} \nabla p \quad \text { weakly in } L^{2}(\Omega)^{3}, \\
\operatorname{div}\left(\mathbf{v}^{D, \varepsilon}-\mathbf{v}^{D}\right) \rightarrow 0 \quad \text { weakly in } L^{2}(\Omega) \text { and } \\
\left(\mathbf{v}^{D, \varepsilon}-\mathbf{v}^{D}\right) \cdot v \rightarrow 0 \quad \text { weakly in } H^{-1 / 2}(\partial \Omega),
\end{gathered}
$$

as $\varepsilon \rightarrow 0$. The above convergence properties allow us to pass to the limit $\varepsilon \rightarrow 0$ in the variational equations (29) and (30). Hence the limit $(\mathbf{u}, p, \vartheta)$ satisfies equations (25) and (26). Furthermore, subtracting equations (30) and (26) and testing the result by $\psi=p^{\varepsilon}-p$ yields

$$
\mathbf{v}^{D, \varepsilon} \rightarrow \mathbf{v}^{D}=-\frac{k}{\eta(\vartheta)} \nabla p \quad \text { strongly in } \quad L^{2}(\Omega) .
$$

Passing to the limit $\varepsilon \rightarrow 0$ in equation (31) is slightly more involved. We use again the coercivity estimates (37).

First notice that, with $C_{\min }:=\frac{1}{a} \frac{K_{S}}{K_{T}} \frac{C_{f}^{2} k}{\eta_{\min }}$, we have for any $s \in \mathbb{R}$

$$
\begin{gathered}
\beta_{0}+\frac{1}{2 a} \frac{K_{S}}{K_{T}} \frac{C_{f}^{2} k}{\eta_{\min }} s^{2}=\frac{1}{2} \frac{C_{\min }}{C_{\max }}\left(\frac{2 C_{\max }}{C_{\min }} \beta_{0}+C_{\max } s^{2}\right) \\
\left.=\frac{1}{2} \frac{C_{\min }}{C_{\max }}\left(\left(\frac{2 C_{\max }}{C_{\min }}-1\right) \beta_{0}+\frac{1}{2} \frac{C_{\min }}{C_{\max }}\left(\beta_{0}+C_{\max } s^{2}\right)\right)>\frac{1}{2} \frac{C_{\min }}{C_{\max }} \beta(s)\right), \quad \text { with A2. }
\end{gathered}
$$


Applying this inequality to (37) yields

$$
\left\|\sqrt{\beta\left(R_{\varepsilon}\left(\vartheta^{\varepsilon}\right)\right)} \nabla \vartheta^{\varepsilon}\right\|_{L^{2}(\Omega)^{m}} \leq C
$$

where $C$ is independent of $\varepsilon$. Then we deduce

$$
\begin{gathered}
R_{\varepsilon}\left(\vartheta^{\varepsilon}\right) \rightarrow \vartheta \quad \text { weakly in } H^{1}(\Omega), \\
\sqrt{\beta\left(R_{\varepsilon}\left(\vartheta^{\varepsilon}\right)\right)} \nabla \vartheta^{\varepsilon} \rightarrow \sqrt{\beta(\vartheta)} \nabla \vartheta \quad \text { weakly in } L^{2}(\Omega)^{3}, \\
\beta\left(R_{\varepsilon}\left(\vartheta^{\varepsilon}\right)\right) \nabla \vartheta^{\varepsilon} \rightarrow \beta(\vartheta) \nabla \vartheta \quad \text { weakly in } L^{3 / 2}(\Omega)^{3}, \\
\int_{\Omega} \mathcal{K}_{\varepsilon} \star \mathbf{v}^{D, \varepsilon} \cdot \nabla R_{\varepsilon}\left(\vartheta^{\varepsilon}\right) \theta d x \rightarrow \int_{\Omega} \mathbf{v}^{D} \cdot \nabla \vartheta \theta d x, \forall \theta \in C(\bar{\Omega}),
\end{gathered}
$$

as $\varepsilon \rightarrow 0$.

Using these results we may pass to the limit $\varepsilon \rightarrow 0$ in equation (31) without difficulty. It completes the proof of Proposition 7.

\subsection{Time discrete Lyapunov functional and stability estimates for the time discretized problem}

To complete the study of the time discrete problem, we need to estimate the behavior of solutions after at least $O(1 / \tau)$ time steps.

In problem (IP), where the time step $\tau$ enters as parameter, one finds after in the first step $\left(\mathbf{u}^{1}, p^{1}, \vartheta^{1}\right)$ from the initial values $\left.(\operatorname{div} \mathbf{u}, p, \vartheta)\right|_{t=0}=\left(\mathcal{E}_{0}, \vartheta_{i n}, p_{\text {in }}\right)$. The idea is to repeat this procedure for an arbitrary number of steps. If $L \in \mathbb{N}, L \leq N=T / \tau$, then $\left(\mathbf{u}^{L}, p^{L}, \vartheta^{L}\right)$ denotes the time discrete approximation of the original quasi-static equations, at $t=t_{L}=L \tau$.

The corresponding free energy at $t=t_{L}$ reads

$$
J^{L}=\int_{\Omega}\left(\mu\left|e\left(\mathbf{u}^{L}\right)\right|^{2}+\frac{1}{2}\left(\lambda\left|\operatorname{div} \mathbf{u}^{L}\right|^{2}+\frac{\left(p^{L}-K_{S} \vartheta^{L}\right)^{2}}{M}+\frac{K_{S}\left(\vartheta^{L}\right)^{2}}{K_{T}}\right)-\mathbf{F}^{L} \cdot \mathbf{u}^{L}\right) d x .
$$

It satisfies the following Lyapunov estimate.

Theorem 8. Under the conditions of Proposition 7 we have for each $L \in \mathbb{N}, L \leq$ $N=T / \tau$, the estimate:

$$
\begin{gathered}
J^{L}+\tau \sum_{j=1}^{L}\left(\int_{\partial \Omega} q^{d, j} p^{j} d S+\int_{\Omega}\left(\frac{\left(\mathbf{F}^{j}-\mathbf{F}^{j-1}\right)}{\tau} \mathbf{u}^{j-1}-Q^{j} p^{j}-H^{j} \vartheta^{j}\right) d x\right)+ \\
\left.\tau \sum_{j=1}^{L} \int_{\Omega}\left(\frac{a k \eta_{\min }}{2} \frac{1}{\eta\left(\vartheta^{j}\right)^{2}}\left|\nabla p^{j}\right|^{2}+\frac{1}{2 a} \frac{k}{C_{\max } \eta_{\min }}\left(\frac{K_{S} C_{f}}{K_{T}}\right)^{2} \beta\left(\vartheta^{j}\right)\right)\left|\nabla \vartheta^{j}\right|^{2}\right) d x \leq J^{0} .
\end{gathered}
$$


Here

$$
J^{0}=\int_{\Omega}\left(\mu\left|e\left(\mathbf{u}^{0}\right)\right|^{2}+\frac{1}{2}\left(\lambda\left|\mathcal{E}^{0}\right|^{2}+\frac{\left(p_{i n}-K_{S} \vartheta_{i n}\right)^{2}}{M}+\frac{K_{S} \vartheta_{i n}^{2}}{K_{T}}\right)-\mathbf{F}^{0} \cdot \mathbf{u}^{0}\right) d x
$$

Proof. We cannot use $\theta=\vartheta$ as test function in the temperature equation (27). However, see Remark 4, this is allowed in equation (31) of the regularized problem $(\mathbf{I P})_{\varepsilon}$.

At time $t=t_{j}$, with $j=1, \ldots, N$, the equations in problem $(\mathbf{I P})_{\varepsilon}$ read

$$
\begin{gathered}
\int_{\Omega} 2 \mu e\left(\mathbf{u}^{\varepsilon, j}\right): e(\xi) d x-\int_{\Omega}\left(b p^{\varepsilon, j}+(1-b) K_{S} \vartheta^{\varepsilon, j}-\lambda \operatorname{div} \mathbf{u}^{\varepsilon, j}\right) \operatorname{div} \xi d x=\int_{\Omega} \mathbf{F}^{j} \xi d x \\
\forall \xi \in H_{0}^{1}(\Omega)^{3} \cdot \\
\int_{\Omega} \partial_{\tau}\left(\frac{p^{\varepsilon, j}-K_{S} \vartheta^{\varepsilon, j}}{M}+b \operatorname{div} \mathbf{u}^{\varepsilon, j}\right) \psi d x+a \int_{\Omega} \frac{k}{\eta\left(\vartheta^{\varepsilon, j}\right)} \nabla p^{\varepsilon, j} \cdot \nabla \psi d x= \\
\int_{\Omega} Q^{j} \psi d x-\int_{\partial \Omega} q^{d, j} \psi d S, \quad \forall \psi \in H^{1}(\Omega) ; \\
\int_{\Omega} \partial_{\tau}\left(a_{0} \vartheta^{\varepsilon, j}-\frac{K_{S}}{M} p^{\varepsilon, j}+(1-b) K_{S} \operatorname{div} \mathbf{u}^{\varepsilon, j}\right) \theta d x+\frac{K_{S} C_{f}}{K_{T}} \int_{\Omega} \mathbf{v}^{D, j, \varepsilon} \cdot \nabla \vartheta^{\varepsilon, j} \theta d x+ \\
\frac{K_{S}}{K_{T}} \int_{\Omega} \beta\left(\vartheta^{\varepsilon, j}\right) \nabla \vartheta^{\varepsilon, j} \cdot \nabla \theta d x=\int_{\Omega} H^{j} \theta d x, \quad \forall \theta \in H^{1}(\Omega)
\end{gathered}
$$

Next, we test (57) with $\xi=\left(\mathbf{u}^{\varepsilon, j}-\mathbf{u}^{\varepsilon, j-1}\right) / \tau$, (58) with $\psi=p^{\varepsilon, j}$ and (59) with $\theta=\vartheta^{j}$. The resulting equalities are added and summed-up with respect to $j$ from $j=1$ to $j=L$. Using the observations

(i) the cross terms containing the pressure, the volume strain and temperature cancel;

(ii)

$$
\sum_{j=1}^{L}\left(2 \mu e\left(\mathbf{u}^{\varepsilon, j}\right): e\left(\mathbf{u}^{\varepsilon, j}-\mathbf{u}^{j-1, \varepsilon}\right) \geq \mu\left|e\left(\mathbf{u}^{\varepsilon, L}\right)\right|^{2}-\mu\left|e\left(\mathbf{u}^{0}\right)\right|^{2}\right.
$$

(iii)

$$
\begin{aligned}
& \sum_{j=1}^{L}\left(\left(b p^{\varepsilon, j}+(1-b) K_{S} \vartheta^{\varepsilon, j}-\lambda \operatorname{div} \mathbf{u}^{\varepsilon, j}\right) \operatorname{div}\left(\mathbf{u}^{\varepsilon, j}-\mathbf{u}^{\varepsilon, j-1}\right)+\right. \\
& \left(\frac{p^{\varepsilon, j}-p^{\varepsilon, j-1}-K_{S}\left(\vartheta^{\varepsilon, j}-\vartheta^{\varepsilon, j-1}\right)}{M}+b \operatorname{div}\left(\mathbf{u}^{\varepsilon, j}-\mathbf{u}^{\varepsilon, j-1}\right)\right) p^{\varepsilon, j}
\end{aligned}
$$




$$
\begin{gathered}
\left.+\left(a_{0}\left(\vartheta^{\varepsilon, j}-\vartheta^{\varepsilon, j-1}\right)-\frac{K_{S}}{M}\left(p^{\varepsilon, j}-p^{\varepsilon, j-1}\right)+(1-b) K_{S} \operatorname{div}\left(\mathbf{u}^{\varepsilon, j}-\mathbf{u}^{\varepsilon, j-1}\right)\right) \vartheta^{\varepsilon, j}\right) \\
\geq \frac{1}{2}\left(\lambda\left|\operatorname{div} \mathbf{u}^{\varepsilon, L}\right|^{2}+\frac{\left(p^{\varepsilon, L}-K_{S} \vartheta^{\varepsilon, L}\right)^{2}}{M}+\frac{K_{S}\left(\vartheta^{\varepsilon, L}\right)^{2}}{K_{T}}\right)-\frac{1}{2}\left(\lambda\left|\operatorname{div} \mathbf{u}^{0}\right|^{2}\right. \\
\left.+\frac{\left(p_{i n}-K_{S} \vartheta_{i n}\right)^{2}}{M}+\frac{K_{S} \vartheta_{i n}^{2}}{K_{T}}\right) ;
\end{gathered}
$$

(iv) directly from inequalities (37) and (50)

$$
\begin{aligned}
& \sum_{j=1}^{L} \int_{\Omega}\left(\frac{a k}{\eta\left(\vartheta^{\varepsilon, j}\right)}\left|\nabla p^{\varepsilon, j}\right|^{2}+\frac{K_{S} C_{f}}{K_{T}} \mathcal{K}_{\varepsilon} \star \mathbf{v}^{D, j, \varepsilon} \cdot \nabla R_{\varepsilon}\left(\vartheta^{\varepsilon, j}\right) \vartheta^{\varepsilon, j}+\frac{K_{S}}{K_{T}} \beta\left(\vartheta^{\varepsilon, j}\right)\left|\nabla \vartheta^{\varepsilon, j}\right|^{2}\right) d x \\
& \geq \frac{1}{2} a k \eta_{\min }\left\|\frac{\nabla p^{\varepsilon, j}}{\eta\left(\vartheta^{\varepsilon, j}\right)}\right\|_{L^{2}(\Omega)^{m}}^{2}+\frac{1}{2 a} \frac{k}{C_{\text {max }} \eta_{\min }}\left(\frac{K_{S} C_{f}}{K_{T}}\right)^{2}\left\|\sqrt{\beta\left(R_{\varepsilon}\left(\vartheta^{\varepsilon, j}\right)\right)} \nabla \vartheta^{\varepsilon, j}\right\|_{L^{2}(\Omega)^{m}}^{2} .
\end{aligned}
$$

$(\mathbf{v})$

$$
\begin{gathered}
\sum_{j=1}^{L} \int_{\Omega}\left(\mathbf{F}^{j}\left(\mathbf{u}^{\varepsilon, j}-\mathbf{u}^{\varepsilon, j-1}\right)+Q^{j} p^{\varepsilon, j}+H^{j} \vartheta^{\varepsilon, j}\right) d x-\sum_{j=1}^{L} \int_{\partial \Omega} q^{d, j} p^{\varepsilon, j} d S= \\
\int_{\Omega}\left(\mathbf{F}^{L} \mathbf{u}^{\varepsilon, L}-\mathbf{F}^{0} \mathbf{u}^{0}\right) d x+\sum_{j=1}^{L} \int_{\Omega}\left(-\left(\mathbf{F}^{j}-\mathbf{F}^{j-1}\right) \mathbf{u}^{\varepsilon, j}+Q^{j} p^{\varepsilon, j}+H^{j} \vartheta^{\varepsilon, j}\right) d x \\
-\sum_{j=1}^{L} \int_{\partial \Omega} q^{d, j} p^{\varepsilon, j} d S,
\end{gathered}
$$

we obtain inequality (56) for $\left(\mathbf{u}^{\varepsilon, j}, p^{\varepsilon, j}, \vartheta^{\varepsilon, j}\right)$. Then using the convergence properties from the proof of Proposition 7, and the convexity of $J^{L}$ and the dissipative term, we conclude that inequality (56) holds for $\left(\mathbf{u}^{j}, p^{j}, \vartheta^{j}\right)$ as well.

Having established existence for the discrete problem (IP) in Proposition 7 and a Lyapunov estimate in Theorem 8, we are in a position to obtain an estimate that is uniform in the time step $\tau$.

Corollary 9. There exists a constant $C>0$ such that

$$
\left\|\mathbf{u}^{L}\right\|_{H^{1}(\Omega)^{3}}^{2}+\left\|p^{L}\right\|_{L^{2}(\Omega)}^{2}+\left\|\vartheta^{L}\right\|_{L^{2}(\Omega)}^{2}+\tau \sum_{j=1}^{L} \int_{\Omega}\left(\left|\nabla p^{j}\right|^{2}+\left|\nabla \vartheta^{j}\right|^{2}\right) d x \leq C,
$$

for all $L$ and $\tau$ such that $1 \leq L \leq N=T / \tau$, with $\tau$ sufficiently small. 


\section{Existence and stability for time discrete problem with thermal convec- tion through heat fluxes, i.e. expression (10)}

The goal of this section is to show that the theory developed in Section 2, applies also to the model, where the heat transport through convection has the dimensionless term $\operatorname{div}\left(C_{f} \mathbf{v}^{D} \vartheta\right)$.

Then system (19)-(21) becomes

$$
\begin{gathered}
-\operatorname{div}\left(2 \mu e\left(\mathbf{u}^{j}\right)+\lambda \operatorname{div} \mathbf{u}^{j} I-b p^{j} \mathbb{I}-(1-b) K_{S} \vartheta^{j} \mathbb{I}\right)=\mathbf{F}^{j}, \\
\partial_{\tau}\left(\frac{p^{j}-K_{S} \vartheta^{j}}{M}+b \operatorname{div} \mathbf{u}^{j}\right)-a \operatorname{div}\left(\frac{k}{\eta\left(\vartheta^{j}\right)} \nabla p^{j}\right)=Q^{j}, \\
\partial_{\tau}\left(a_{0} \vartheta^{j}-\frac{K_{S}}{M} p^{j}+(1-b) K_{S} \operatorname{div} \mathbf{u}^{j}\right)+\frac{K_{S}}{K_{T}} \operatorname{div}\left(C_{f} \mathbf{v}^{D, j} \vartheta^{j}-\beta\left(\vartheta^{j}\right) \nabla \vartheta^{j}\right)=H^{j},
\end{gathered}
$$

with $\mathbf{v}^{D, j}$ given by (22).

The variational (or virtual work) formulation of equations (61)-(63), (22), subject to conditions (23) and (24), reads:

Problem (IPdiv) (incremental problem): $\quad$ Let $W:=H_{0}^{1}(\Omega)^{m} \times H^{1}(\Omega)^{2}$. Then for given $(\mathbf{U}, \pi, \Theta) \in W$, find $(\mathbf{u}, p, \vartheta) \in W$, with $\sqrt{\beta(\vartheta)} \nabla \vartheta \in L^{2}(\Omega)^{m}$, such that variational equations (25)-(26) are satisfied and

$$
\begin{gathered}
\int_{\Omega} \partial_{\tau}\left(a_{0} \vartheta-\frac{K_{S}}{M} p+(1-b) K_{S} \operatorname{div} \mathbf{u}\right) \theta d x+\frac{K_{S}}{K_{T}} \int_{\Omega}\left(-C_{f} \mathbf{v}^{D} \vartheta+\beta(\vartheta) \nabla \vartheta\right) \cdot \nabla \theta d x \\
+\frac{K_{S} C_{f}}{K_{T}} \int_{\partial \Omega} q^{d} \vartheta \theta d S=\int_{\Omega} H \theta d x, \quad \forall \theta \in W^{1, r}(\Omega), r>m
\end{gathered}
$$

Problem (IPdiv) meets the same difficulties as problem (IP). Accordingly we introduce the regularized incremental problem

Problem $(\mathbf{I P d i v})_{\varepsilon}: \quad$ Given $(\mathbf{U}, \pi, \Theta) \in W$, find $\left(\mathbf{u}^{\varepsilon}, p^{\varepsilon}, \vartheta^{\varepsilon}\right) \in W$ such that variational equations (29)-(30) are satisfied and

$$
\begin{gathered}
\int_{\Omega} \partial_{\tau}\left(a_{0} \vartheta^{\varepsilon}-\frac{K_{S}}{M} p^{\varepsilon}+K_{S}(1-b) \operatorname{divu}^{\varepsilon}\right) \theta d x+\frac{K_{S}}{K_{T}} \int_{\Omega}\left(-C_{f} \mathcal{K}_{\varepsilon} \star \mathbf{v}^{D, \varepsilon} R_{\varepsilon}\left(\vartheta^{\varepsilon}\right)+\right. \\
\left.\beta\left(R_{\varepsilon}\left(\vartheta^{\varepsilon}\right)\right) \nabla \vartheta^{\varepsilon}\right) \cdot \nabla \theta d x+\frac{K_{S} C_{f}}{K_{T}} \int_{\partial \Omega} q^{d} R_{\varepsilon}\left(\vartheta^{\varepsilon}\right) \theta d S=\int_{\Omega} H \theta d x, \forall \theta \in H^{1}(\Omega) .
\end{gathered}
$$


Proposition 10. Let $\varepsilon>0$ be a small positive constant and let assumptions $A 1-3$, where we take $q^{d} \in C\left([0, T] ; L^{\infty}(\partial \Omega)\right)$, be satisfied. Furthermore, let

$$
\frac{1}{\tau} \geq 2 C_{f} C_{t r}(\Omega)\left\|q^{\delta}\right\|_{L^{\infty}(\partial \Omega)}
$$

where $C_{t r}(\Omega)$ is the constant in the trace inequality between $W^{1,1}(\Omega)$ and $L^{1}(\partial \Omega)$. Then Problem (IPdiv) $)_{\varepsilon}$ admits at least one solution $\left(\mathbf{u}^{\varepsilon}, p^{\varepsilon}, \vartheta^{\varepsilon}\right) \in W$.

Proof. The proof is identical to the proof of Proposition 5, except for the coercivity estimate.

Taking $\xi=\mathbf{u}^{\varepsilon}, \psi=p^{\varepsilon}$ and $\theta=\vartheta^{\varepsilon}$ in (29)-(30), (65) yields

$$
\begin{gathered}
\left\langle\mathcal{A}\left(\mathbf{u}^{\varepsilon}, p^{\varepsilon}, \vartheta^{\varepsilon}\right),\left(\mathbf{u}^{\varepsilon}, p^{\varepsilon}, \vartheta^{\varepsilon}\right)\right\rangle=\frac{2 \mu}{\tau} \int_{\Omega}\left|e\left(\mathbf{u}^{\varepsilon}\right)\right|^{2} d x+\frac{1}{\tau} \int_{\Omega}\left(\lambda\left|\operatorname{div} \mathbf{u}^{\varepsilon}\right|^{2}+\frac{\left(p^{\varepsilon}-K_{S} \vartheta^{\varepsilon}\right)^{2}}{M}\right. \\
\left.+\frac{K_{S}\left(\vartheta^{\varepsilon}\right)^{2}}{K_{T}}\right) d x+a \int_{\Omega} \frac{k}{\eta\left(\vartheta^{\varepsilon}\right)}\left|\nabla p^{\varepsilon}\right|^{2} d x-\frac{K_{S} C_{f}}{K_{T}} \int_{\Omega} \mathcal{K}_{\varepsilon} \star \mathbf{v}^{D, \varepsilon} \cdot \nabla \vartheta^{\varepsilon} R_{\varepsilon}\left(\vartheta^{\varepsilon}\right) d x \\
+\frac{K_{S}}{K_{T}} \int_{\Omega} \beta\left(R_{\varepsilon}\left(\vartheta^{\varepsilon}\right)\right)\left|\nabla \vartheta^{\varepsilon}\right|^{2} d x+\frac{K_{S} C_{f}}{K_{T}} \int_{\partial \Omega} q^{d} R_{\varepsilon}\left(\vartheta^{\varepsilon}\right) \vartheta^{\varepsilon} d S
\end{gathered}
$$

with $\mathbf{v}^{D, \varepsilon}=-k \nabla p^{\varepsilon} / \eta\left(\vartheta^{\varepsilon}\right)$.

The 4th integral containing the Darcy discharge is estimated in exactly same way as the equivalent integral in the coercivity estimate of the proof of Proposition 5. We only need to estimate the boundary integral in (67).

Using the trace theorem for the space $W^{1,1}(\Omega)$ yields

$$
\begin{gathered}
\left|\int_{\partial \Omega} q^{d} R_{\varepsilon}\left(\vartheta^{\varepsilon}\right) \vartheta^{\varepsilon} d S\right| \leq\left\|q^{d}\right\|_{L^{\infty}(\Omega)}\left\|R_{\varepsilon}\left(\vartheta^{\varepsilon}\right) \vartheta^{\varepsilon}\right\|_{L^{1}(\partial \Omega)} \leq\left\|q^{d}\right\|_{L^{\infty}(\Omega)} C_{t r} \int_{\Omega}\left(\left|R_{\varepsilon}\left(\vartheta^{\varepsilon}\right) \vartheta^{\varepsilon}\right|\right. \\
\left.+\left|\nabla\left(R_{\varepsilon}\left(\vartheta^{\varepsilon}\right) \vartheta^{\varepsilon}\right)\right|\right) d x \leq\left\|q^{d}\right\|_{L^{\infty}(\Omega)} C_{t r}(\Omega)\left(\int_{\Omega}\left(\vartheta^{\varepsilon}\right)^{2} d x+2 \int_{\Omega}\left|R_{\varepsilon}\left(\vartheta^{\varepsilon}\right) \| \nabla \vartheta^{\varepsilon}\right| d x\right) \leq \\
\left\|q^{d}\right\|_{L^{\infty}(\Omega)} C_{t r}(\Omega)\left(\int_{\Omega}\left(\vartheta^{\varepsilon}\right)^{2} d x+2 \int_{\Omega} \frac{\sqrt{\beta\left(R_{\varepsilon}\left(\vartheta^{\varepsilon}\right)\right)}}{\sqrt{C_{\text {min }}}}\left|\nabla \vartheta^{\varepsilon}\right| d x\right)
\end{gathered}
$$

The term in the last inequality containing $\int_{\Omega} \sqrt{\beta\left(R_{\varepsilon}\left(\vartheta^{\varepsilon}\right)\right)}\left|\nabla \vartheta^{\varepsilon}\right| d x$ is controlled by the $L^{2}$-norm squared of $\sqrt{\beta\left(R_{\varepsilon}\left(\vartheta^{\varepsilon}\right)\right)} \nabla \vartheta^{\varepsilon}$ in $(67)$ and the term containing $\int_{\Omega}\left(\vartheta^{\varepsilon}\right)^{2} d x$ can be collected with other $\int_{\Omega}\left(\vartheta^{\varepsilon}\right)^{2} d x$ terms in (67). With the coefficient $K_{S} / K_{T}$ multiplying $\vartheta^{2}$, this yields

$$
\frac{K_{S}}{K_{T}}\left(\frac{1}{\tau}-C_{f} C_{t r}(\Omega)\left\|q^{d}\right\|_{L^{\infty}(\partial \Omega)}\right) \int_{\Omega}\left(\vartheta^{\varepsilon}\right)^{2} d x .
$$


Hence condition (66) on the time step $\tau$ implies the coercivity.

Next, $q^{d} \in C\left([0, T] ; L^{\infty}(\partial \Omega)\right)$ and adding (66) to the assumptions of Proposition 7 , gives us, with an identical proof, the analogue for Problem (IPdiv):

Proposition 11. Let the conditions of Proposition 10 be satisfied. Then Problem (IPdiv) admits at least one solution $(\mathbf{u}, p, \vartheta) \in W$, such that $\sqrt{\beta(\vartheta)} \nabla \vartheta \in L^{2}(\Omega)^{3}$.

The results from the time discrete Lyapunov functional from Section 2.2 are again valid, with a minor change in the dissipative part. We omit the details.

\section{Numerical examples}

In this section, we confirm our theoretical results with several numerical tests. We use the open-source finite element library deal.II [1] and specifically an adaptation of the multiphysics template [22].

\subsection{Solution algorithms and discretization}

The discretization of the variational system (25),(26), and (27) is undertaken in a monolithic fashion in which the three equations are solved all-at-once. The technique and a corresponding implementation are discussed for a similar-type threeequation-problem in [22]. For temporal discretization a backward Euler scheme is used as usually done for porous media applications. Spatial discretization is based on a Galerkin finite element scheme. We introduce $H^{1}$ conforming discrete spaces (see e.g., [4]), which consist of continuous bilinear functions $Q_{1}^{c}$. The temperature equation is semi-linear due to the term $\nabla p^{j} \cdot \nabla \vartheta^{j}$. For high Péclet numbers this transport term would require upwinding. However, Assumption A2 on $\beta(\vartheta)$ (see (16)), limits the effect of the Péclet number. Hence we use a Newton linearization (with a tolerance of $T O L_{N e w}=10^{-9}$ ) for which we again refer to [22]. For more comments on the numerical treatment of this term we refer to [16]. All other couplings are linear. Therefore, we have a slightly nonlinear behavior for which Newton's method converges in a few steps (in the below examples, we observe $2-3$ Newton steps).

\subsection{Configuration and parameters}

We work on the domain $\Omega=(0,1)^{2}$. The parameters are taken from the numerical section in [7] and given in Table 2 .

The domain is decomposed into quadrilaterals and are three times uniformly refined. In some tests, we perform mesh refinement studies and work on four, five, six, and seven times refined meshes. We compute $N$ time steps with the time step size $\Delta t=1$, i.e., the final time is $t_{N}$. 


\begin{tabular}{llr}
\hline \hline SYMBOL & QUANTITY & PARAMETER \\
\hline$\eta$ & dimensionless viscosity & 1 \\
\hline$\Phi$ & porosity & 0.082 \\
\hline$b$ & Biot's coefficient & 0.95 \\
\hline$\mu$ & 1st Lamé-Gassmann coefficient & 0.656 \\
\hline$\lambda$ & 2nd Lamé - Gassmann coefficient & 0.429 \\
\hline $1 / M$ & compressibility coefficient & 0.183 \\
\hline$k$ & scalar permeability & $1.77 \cdot 10^{-4}$ \\
\hline$K_{S}$ & solid expansion coefficient & $5 \cdot 10^{-2}$ \\
\hline$K_{T}$ & thermoelastic coupling parameter & $3 \cdot 10^{-4}$ \\
\hline$a_{0}$ & $=K_{S} / K_{T}+K_{S}^{2} / M$ & 166.7 \\
\hline$\beta_{0}$ & thermal diffusivity & 0.98 \\
\hline \hline
\end{tabular}

Table 2: Data description

For simplicity we take a constant viscosity $\eta$ and a constant thermal diffusivity $\beta(\vartheta)=\beta_{0}$. The values of $\vartheta$ are not large and $C_{\text {min }} \vartheta^{2}$ is negligible. Hence the effects of the nonlinearity in $\beta$ are not visible.

\subsection{Example 1: Program verification with manufactured solutions}

In this first numerical test, we construct manufactured solutions and compare them to the numerically obtained values.

\subsubsection{Initial and boundary conditions}

As initial conditions, we set:

$$
\begin{gathered}
\left.p\right|_{t=0}=p_{0}=x-y \quad \text { in } \Omega \\
\left.\vartheta\right|_{t=0}=0 \quad \text { in } \Omega .
\end{gathered}
$$

As boundary conditions, we use:

$$
\begin{aligned}
\mathbf{u} & =(0,0)^{T} \quad \text { on } \partial \Omega \times(0, T) \\
\mathbf{v}^{D} \cdot \nu=-k(1,-1)^{T} \nu & =q^{d, j} \quad \text { on } \partial \Omega \times(0, T) \\
\beta \nabla \vartheta \cdot \nu & =0 \quad \text { on } \partial \Omega \times(0, T) .
\end{aligned}
$$

\subsubsection{Manufactured solutions and resulting right hand sides}

For the manufactured solutions $p$ and $\vartheta$, the right hand side in the momentum equation will be

$$
F=-\mu \Delta \mathbf{u}+\nabla\left(b p+K_{s}(1-b) \vartheta-(\lambda+\mu) \operatorname{div} \mathbf{u}\right)
$$


where

$$
\mathbf{u}=\bar{u}(x-1)^{2} x^{2}(y-1)^{2} y^{2} *(1,1)^{T}
$$

with $\bar{u}=0.1$.

Next, in order to have limited in time source terms for the pressure and temperature equations, we introduce time cut-off terms for $p$ and $\theta$ such that for $t>c_{u p}$ and $t<c_{\text {low }}$ with $c_{u p}=40$ and $c_{\text {low }}=10$ the manufactured right hand side is independent of the time $t$. It guarantees that the time evolution starts at $t=c_{l o w}$ and the solution will stabilize exponentially fast to a stationary solution with $\nabla \vartheta=0$ for $t>c_{u p}$.

Such a setting allows us to simulate a situation with concentrated point heat sources, which can represent e.g. a buried nuclear waste, and with water coming from the outer boundary and through production and injection wells. With this goal in our mind, we use for the pressure and temperature the following manufactured terms simulating point sources:

We chose the radius $r=0.1$ and the middle point $\left(x_{1}, y_{1}\right)=(0.5,0.5)$ for point source 1 and $\left(x_{2}, y_{2}\right)=(0.7,0.3)$ for point source 2 . For the pressure, we take the function

$$
\begin{aligned}
p= & x-y+P_{0}\left(t-c_{\text {low }}\right)_{+}\left(c_{u p}-t\right)_{+} \cdot\left(\exp \left(\frac{-r^{2}}{r^{2}-\left(x-x_{1}\right)^{2}-\left(y-y_{1}\right)^{2}}\right) \chi_{B\left(x_{1}, r\right)}\right. \\
& \left.+\exp \left(\frac{-r^{2}}{r^{2}-\left(x-x_{2}\right)^{2}-\left(y-y_{2}\right)^{2}}\right) \chi_{B\left(x_{2}, r\right)}\right) .
\end{aligned}
$$

Here, $P_{0}=1$. Clearly, for $t>c_{u p}$ and $t<c_{\text {low }}$, the gradient of $p$ is given by:

$$
\nabla p=(1,-1)^{T} \text { for } t>c_{\text {up }}, t<c_{\text {low }} .
$$

For $c_{\text {low }} \leq t \leq c_{\text {up }}$, we have

$$
\begin{aligned}
\nabla p= & (1,-1)^{T}-2 P_{0}\left(t-c_{\text {low }}\right)_{+}\left(c_{\text {up }}-t\right)_{+} \\
& \cdot\left(\exp \left(\frac{-r^{2}}{r^{2}-\left(x-x_{1}\right)^{2}-\left(y-y_{1}\right)^{2}}\right) \chi_{B\left(x_{1}, r\right)} * \frac{r^{2}\left(x-x_{1}, y-y_{1}\right)^{T}}{\left(r^{2}-\left(x-x_{1}\right)^{2}-\left(y-y_{1}\right)^{2}\right)^{2}}\right. \\
& \left.\quad+\exp \left(\frac{-r^{2}}{r^{2}-\left(x-x_{2}\right)^{2}-\left(y-y_{2}\right)^{2}}\right) \chi_{B\left(x_{2}, r\right)} * \frac{r^{2}\left(x-x_{2}, y-y_{2}\right)^{T}}{\left(r^{2}-\left(x-x_{2}\right)^{2}-\left(y-y_{2}\right)^{2}\right)^{2}}\right)
\end{aligned}
$$

We infer from these constructions that for $t>c_{u p}$ and $t<c_{l o w}$ we should have

$$
\mathbf{v}^{D}=-\frac{k}{\eta} \nabla p=-k(1,-1)^{T} \approx 10^{-4}(1,-1)^{T} .
$$

We use this expression to check our Darcy velocities in our numerical simulations below. 
For the temperature, we propose a very similar construction as for the pressures:

$$
\begin{aligned}
\vartheta & =\vartheta_{0}+\Theta_{0}\left(t-c_{\text {low }}\right)_{+}\left(c_{u p}-t\right)_{+}\left(\exp \left(\frac{-r^{2}}{r^{2}-\left(x-x_{1}\right)^{2}-\left(y-y_{1}\right)^{2}}\right) \chi_{B\left(x_{1}, r\right)}\right. \\
& \left.+\exp \left(\frac{-r^{2}}{r^{2}-\left(x-x_{2}\right)^{2}-\left(y-y_{2}\right)^{2}}\right) \chi_{B\left(x_{2}, r\right)}\right),
\end{aligned}
$$

with $\Theta_{0}=0.1$ and $\vartheta_{0}=0$. With this, we have

$$
\vartheta=\vartheta_{0}=0 \text { for } t>c_{\text {up }} \text { and } t<c_{\text {low }} .
$$

These formulas allow us to calculate the right hand sides for $Q$ and $H$ :

$$
\begin{aligned}
& Q=\partial_{t}\left(\frac{p-K_{s} \vartheta}{M}\right)-\frac{\tau_{c}}{\tau_{T}} \operatorname{div}(k \nabla p) \\
& H=\partial_{t}\left(a_{0} \vartheta-\frac{K_{s}}{M} p\right)+\frac{K_{s} C_{f}}{K_{T}} \mathbf{v}^{D} \cdot \nabla \vartheta-\frac{K_{s}}{K_{T}} \operatorname{div}(\beta \nabla \vartheta) .
\end{aligned}
$$

Clearly, with all constructions and cut-off times, we have

$$
H=0 \text { for } t>c_{u p} \text { and } t<c_{\text {low }} .
$$

For time $t \in\left(c_{\text {low }}, c_{\text {up }}\right)$, the source terms $Q$ and $H$ are large, due to the steep derivatives of the concentrated pics in $p$ and $\vartheta$. It would drive the whole system and the final temperature will take a different value from the initial one.

\subsubsection{Numerical simulations with one point source}

We plug-in the previous terms into the system (25),(26), and (27) and solve numerically as described above. Graphical results for $t=30$ and $t=100$ are shown in the Figure 1 and 2. The evolution of several quantities of interest are shown in Figure 3. Therein, we observe a very good agreement of our theoretically predicted Darcy velocities.

Table 3: Norm $\left\|p_{h}(t)-p(t)\right\|_{L^{2}}$ at $t=100$ on different spatial meshes, where $p_{h}$ is the numerical solution.

\begin{tabular}{|l|l|}
\hline$h$ & $\left\|p_{h}(t)-p(t)\right\|_{L^{2}}$ \\
\hline $1.76777 \mathrm{e}-01$ & $1.70216 \mathrm{e}-01$ \\
$8.83883 \mathrm{e}-02$ & $1.74386 \mathrm{e}-01$ \\
$4.41942 \mathrm{e}-02$ & $5.94341 \mathrm{e}-02$ \\
\hline
\end{tabular}



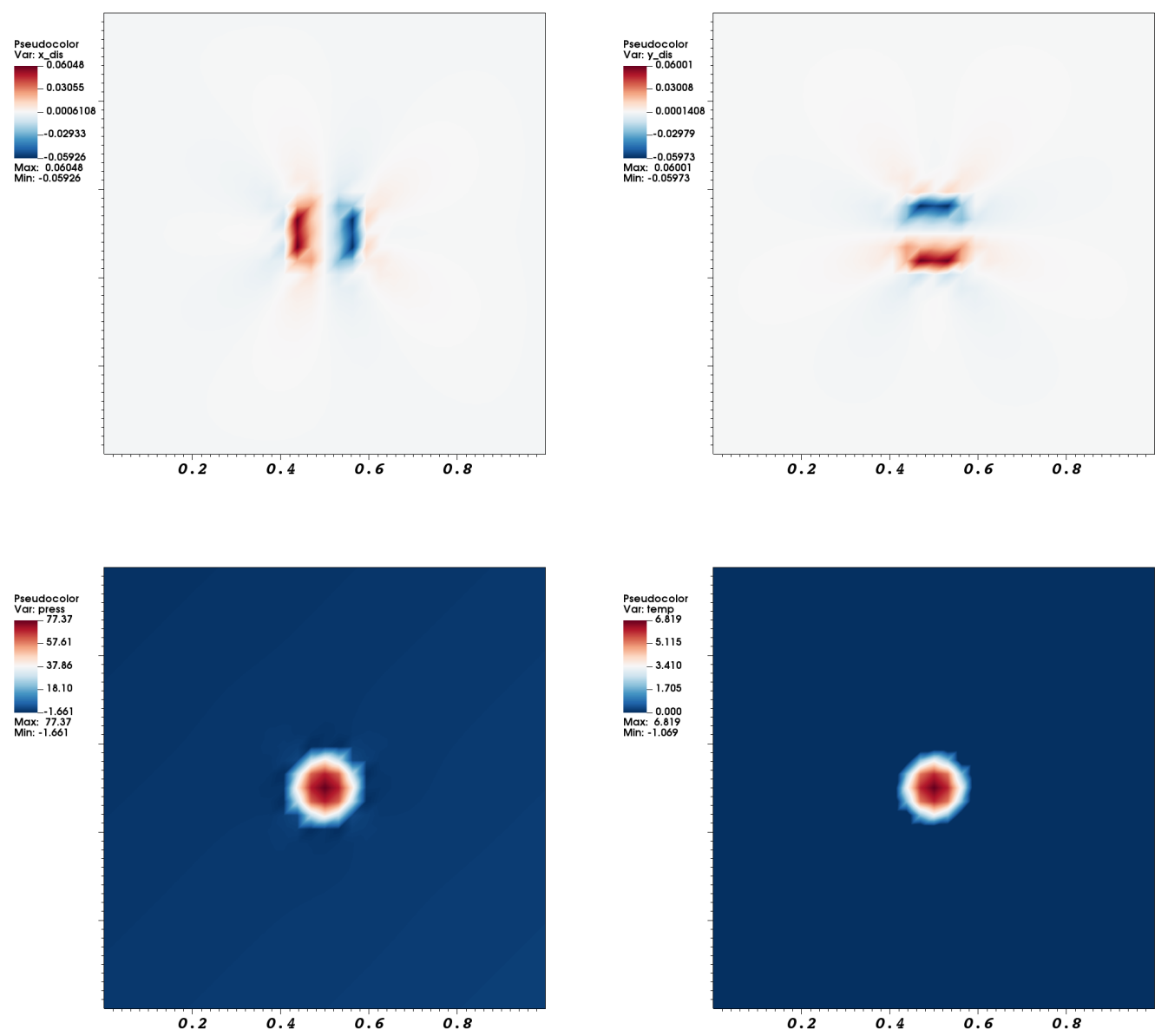

Figure 1: Example 1 (manufactured solution). Going from top left to bottom right: $\mathbf{u}_{x}, \mathbf{u}_{y}, p, \vartheta$ at $t=30 s$ with $N=30$. 

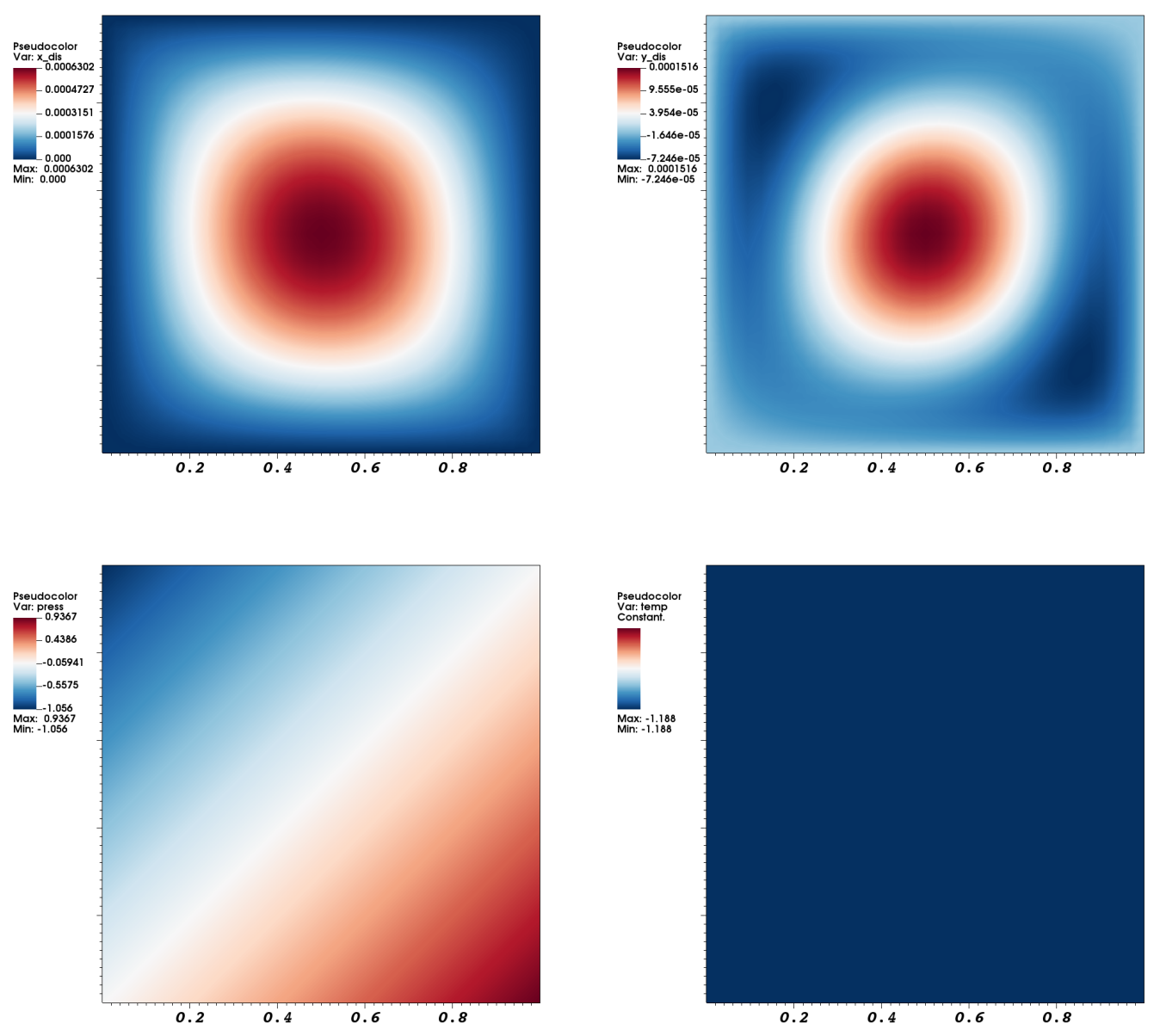

Figure 2: Example 1 (manufactured solution). Going from top left to bottom right: $\mathbf{u}_{x}, \mathbf{u}_{y}, p, \vartheta$ at $t=100 \mathrm{~s}$ with $N=100$. The temperature $\vartheta$ stabilized to a constant values $\vartheta=-1.188 \neq 0$. 

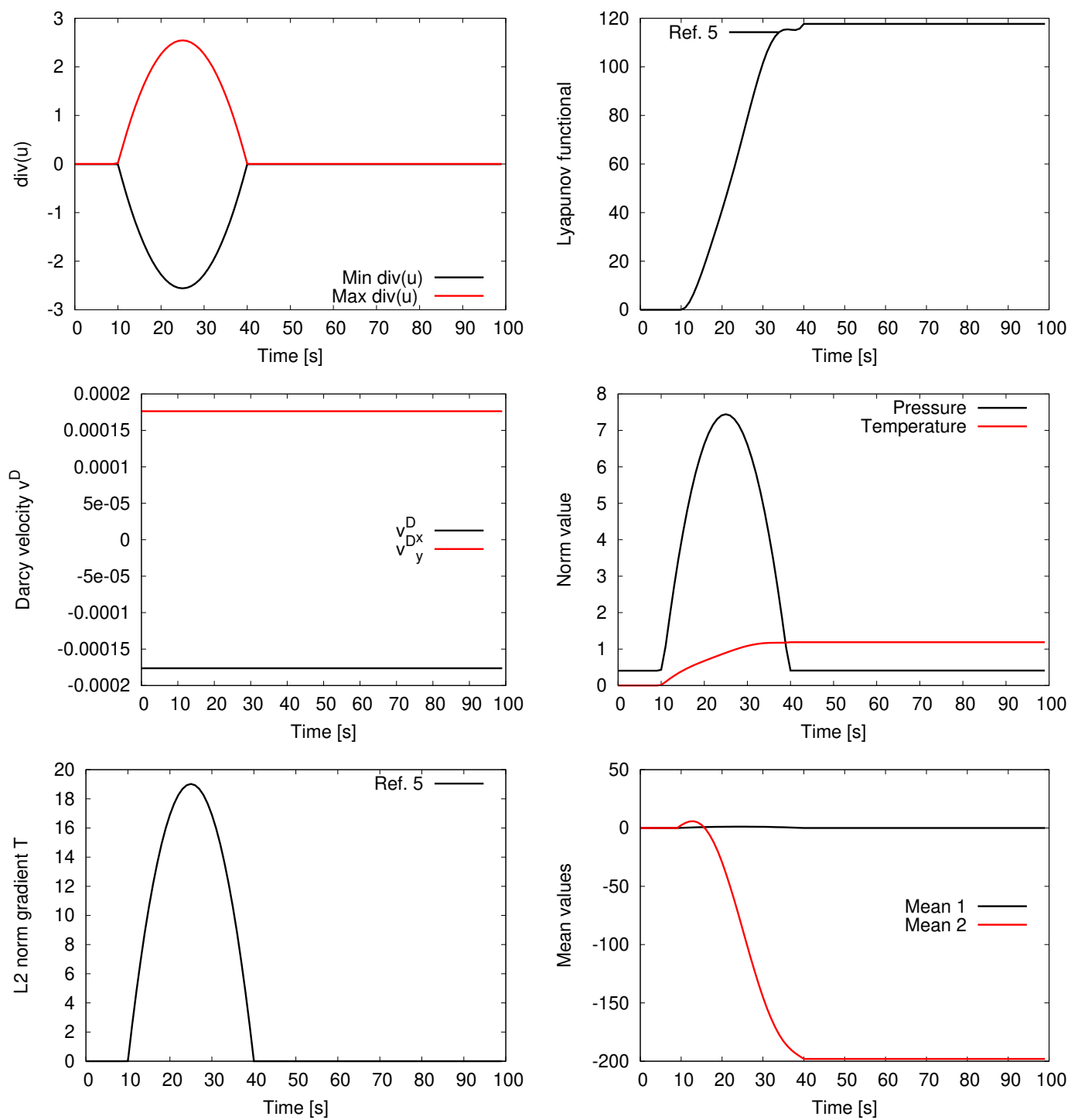

Figure 3: Example 1 (manufactured solution; one point source). Evolution over divu, the Lyapunov functional, the Darcy velocities $\mathbf{v}^{D}$ and the pressure and temperature $L^{2}$ norms. Furthermore, we plot the $L^{2}$ norm of the temperature gradient and two mean values. Specifically, for the Darcy velocities, we observe a very good agreement as predicted in our manufactured solutions. The Mean 1 corresponds to $\left.\int_{\Omega}\left(p-K_{S} \vartheta\right)\right)(t) d x$ (the mean of the fluid content multiplies by $M$ ) and it is conserved for $t>c_{u p}$ and equal to zero. The Mean 2 corresponds to $\int_{\Omega}\left(a_{0} \vartheta-\frac{K_{S}}{M} p\right)(t) d x$ and it is conserved in time as well. The Lyapunov functional stabilized quickly for $t>c_{u p}$ to a constant value, confirming our stability results. 


\subsubsection{Numerical simulations with two point sources}

We now present a simulation with both point sources for the pressures and temperatures. Graphical solution plots are provided in Figure 4 and the evolution of the quantities of interest is shown in Figure 5.
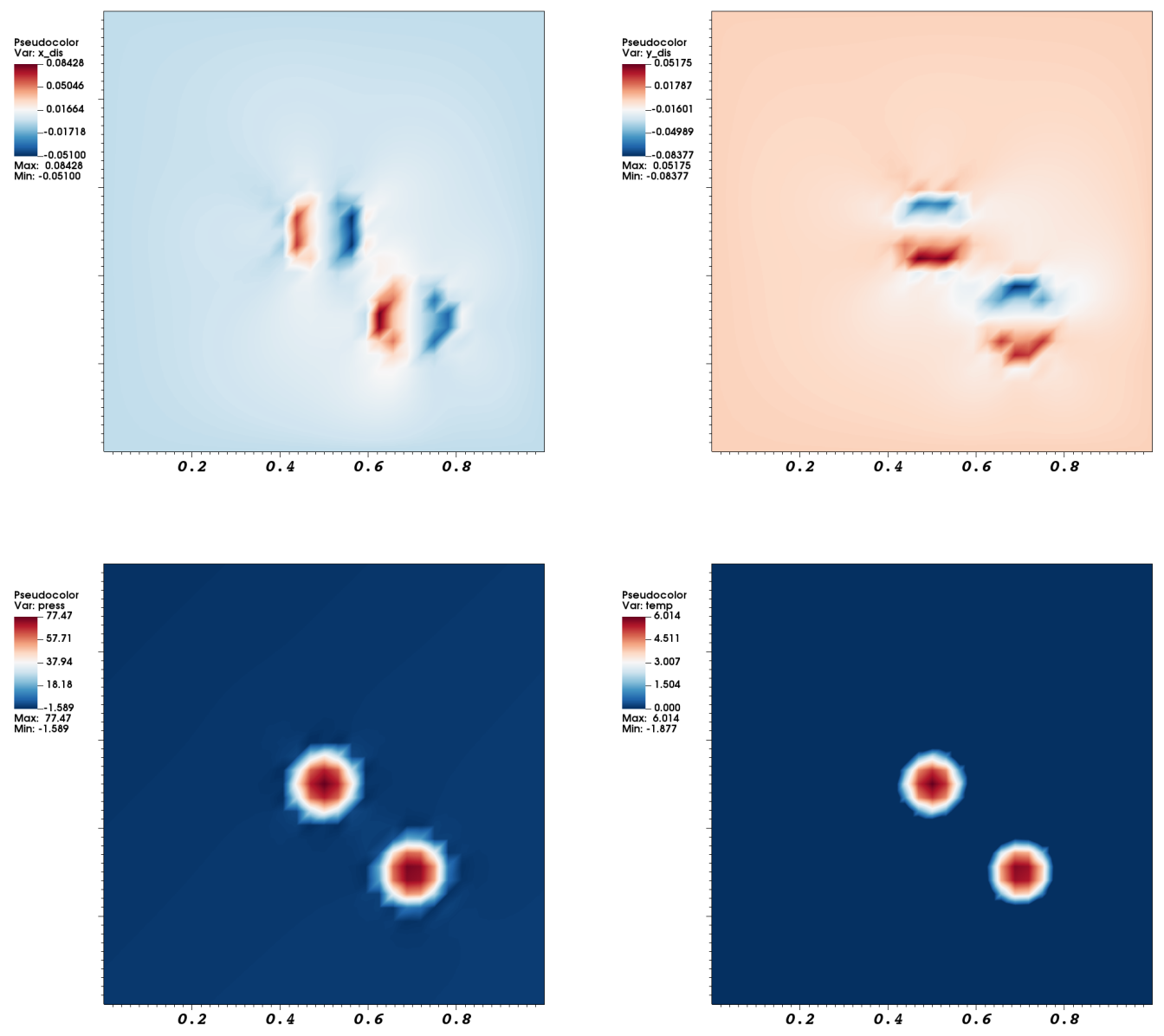

Figure 4: Example 1 (manufactured solution; two point sources). Going from top left to bottom right: $\mathbf{u}_{x}, \mathbf{u}_{y}, p, \vartheta$ at $t=30 \mathrm{~s}$ with $N=30$. We only plot $t=30 \mathrm{~s}$ because $t=100 \mathrm{~s}$ is very similar to the previous numerical test. 

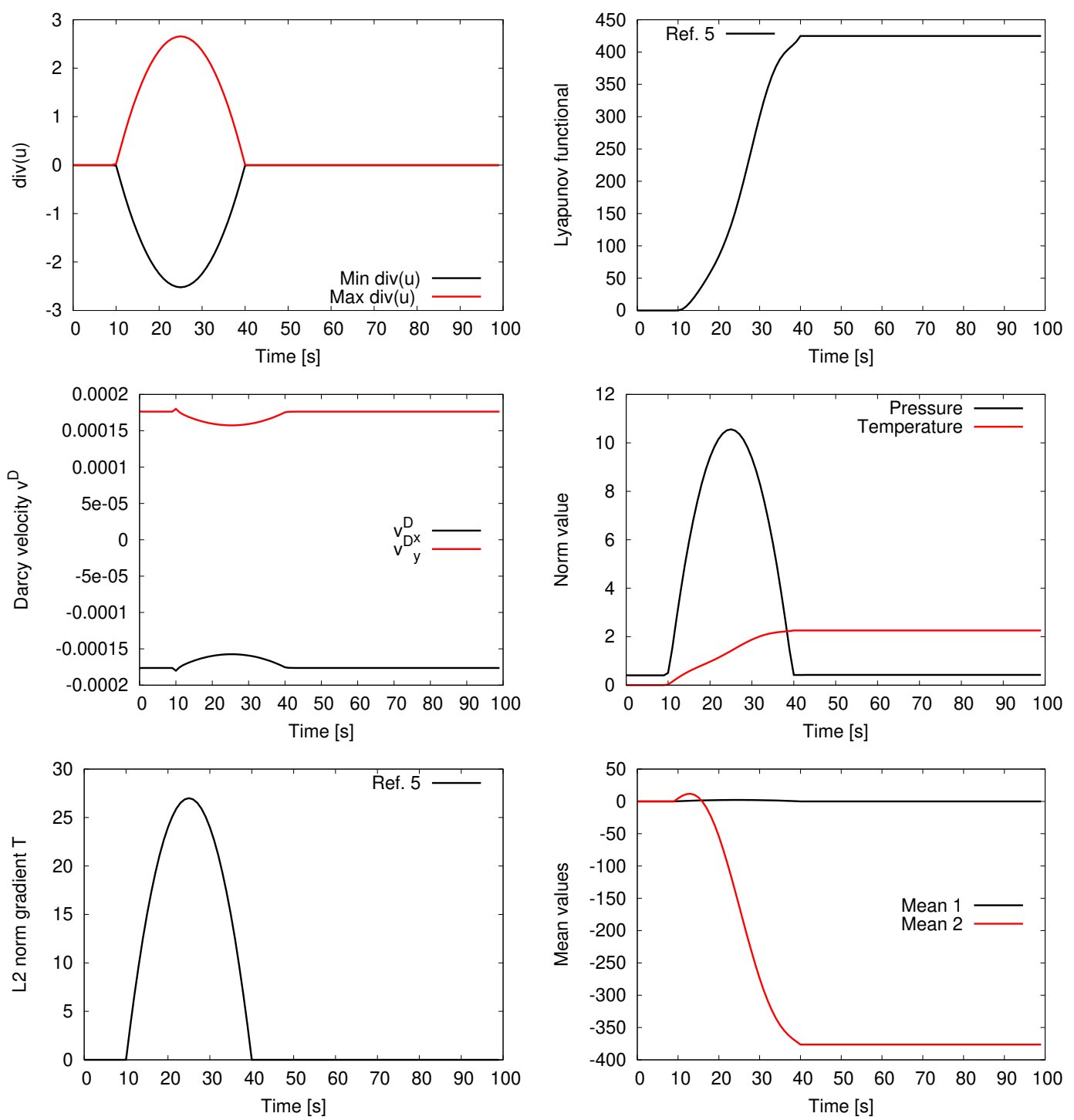

Figure 5: Example 1 (manufactured solution; two point sources). Evolution over divu, the Lyapunov functional, the Darcy velocities $\mathbf{v}^{D}$ and the pressure and temperature $L^{2}$ norms. Furthermore, we plot the $L^{2}$ norm of the temperature gradient and two mean values. Specifically, for the Darcy velocities, we observe a very good agreement as predicted in our manufactured solutions. All the observations from Fig. 3, apply here as well.

\subsection{Example 2: A given right hand side $F^{j}$ for the displacements}

In this numerical test, the right hand side of the displacement equation is prescribed:

$$
F^{j}=(1,1)^{T}, \quad j=0, \ldots N
$$


with $N=10$. Other source terms are set zero, i.e. $Q^{j}=H^{j}=0$ for $j=1, \ldots, N$.. Further we set

$$
\mathbf{u}^{j}=0, \quad \mathbf{v}^{D, j} \cdot \boldsymbol{v}=0 \quad \text { and } \quad \beta(\vartheta) \nabla \vartheta \cdot v=0 \quad \text { along } \partial \Omega, \text { for } j=1, \ldots, N
$$

and initially we take

$$
\left.(\mathbf{u}, p, \vartheta)\right|_{t=0}=0, \quad \text { in } \Omega .
$$

We compute the solutions on three different meshes in order to study the computational stability with respect to mesh refinement. Our findings are shown in the Figures 6 and 7.
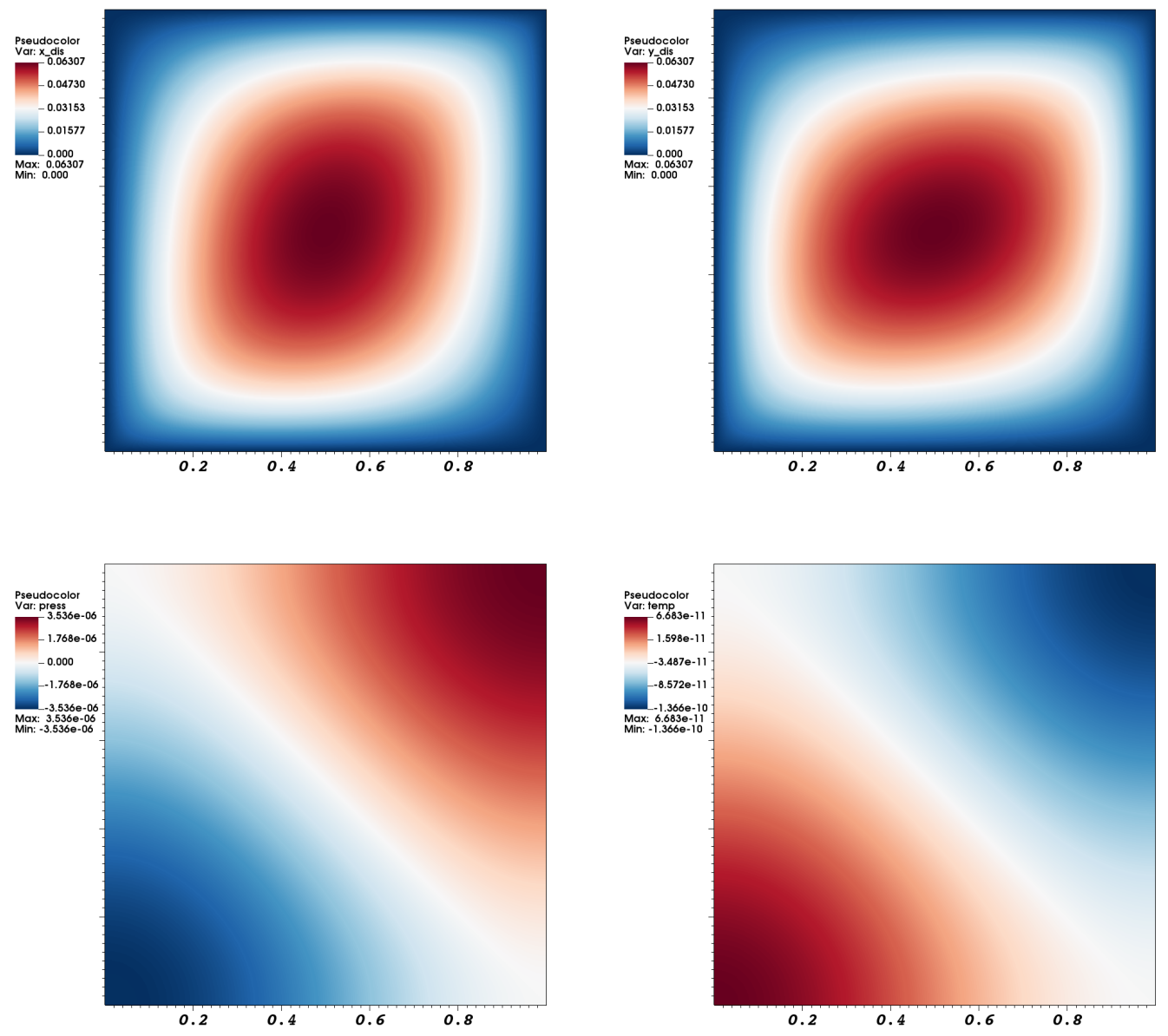

Figure 6: Example 2 (volume force in the displacement equation). Going from top left to bottom right: $\mathbf{u}_{x}, \mathbf{u}_{y}, p, \vartheta$ at $t=10 s$ with $N=10$. 

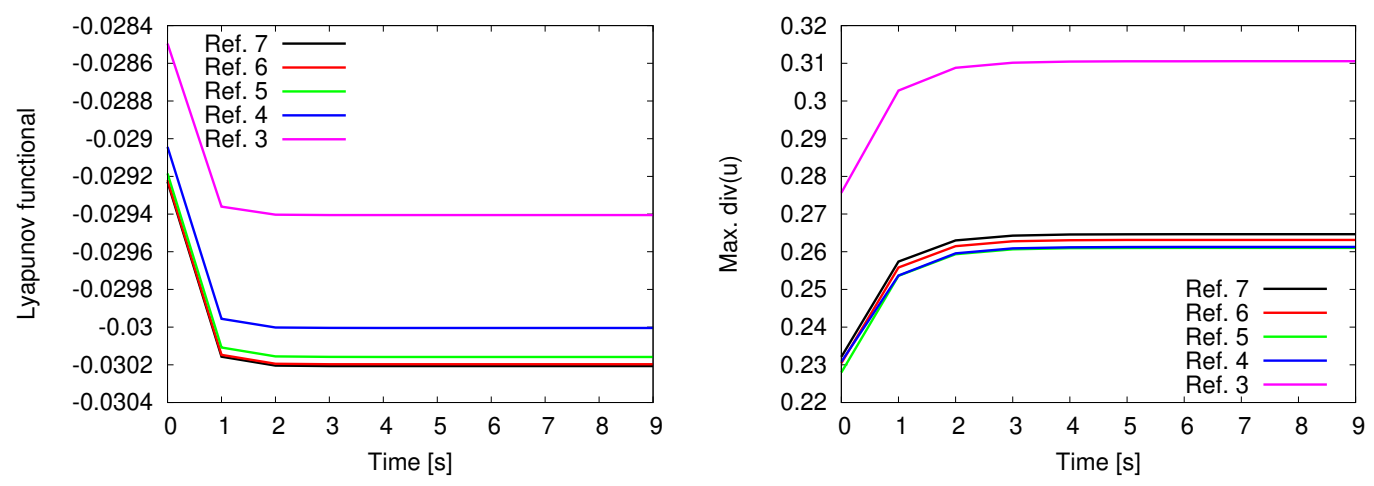

Figure 7: Example 2 (volume force in the displacement equation). Evolution of the Lyapunov functional $J^{m}$ and the maximum divu.

\subsection{Example 3: A source term $Q^{j}$ in the pressure equation}

In this numerical test, a time-dependent pressure source term is prescribed:

$$
Q=t * \exp \left(-f\left(\left(x-x_{c}\right)^{2}+\left(y-y_{c}\right)^{2}\right)\right), \quad x_{c}=y_{c}=0.5
$$

with a parameter $f=10, t=t_{j}, j=0, \ldots, N$ and with $N=100$. For the other source terms, we use $F^{j}=H^{j}=0$. The boundary and initial values are chosen as in Example 2. We compute the solutions on three different meshes in order to study the computational stability with respect to mesh refinement. In Figure 8, we observe the final states of the three fields $\left(\mathbf{u}_{x}, \mathbf{u}_{y}\right), p$ and $\vartheta$. In Figure 9, the Lyapunov functional and the maximum of divu are displayed for three different meshes. Here, we observe qualitative convergence under spatial mesh refinement. Our results are plotted in the Figures 8 and 9 . 

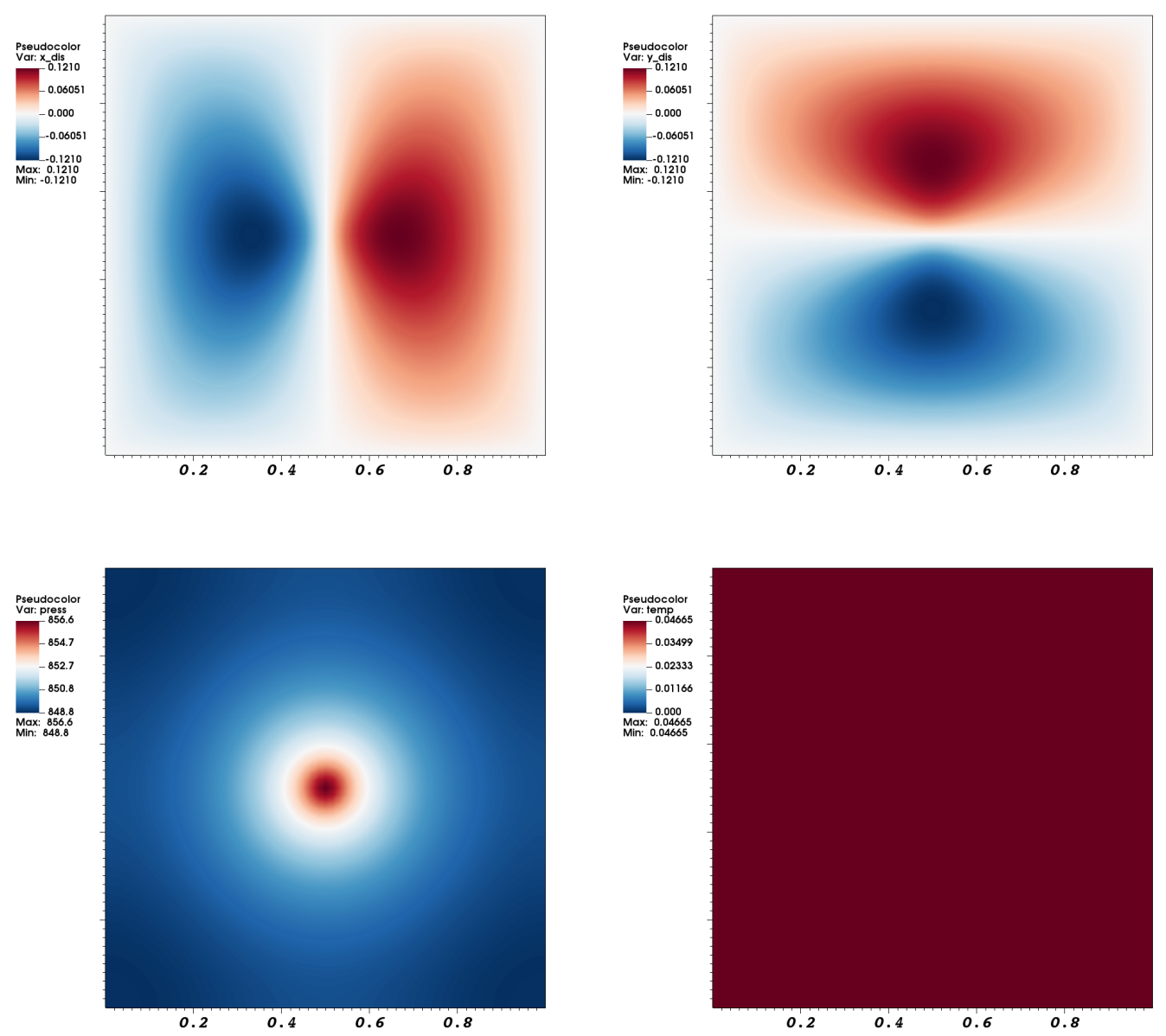

Figure 8: Example 3 (pressure source). Going from top left to bottom right: $\mathbf{u}_{x}, \mathbf{u}_{y}, p, \vartheta$ at $t=100 \mathrm{~s}$ with $N=100$. 

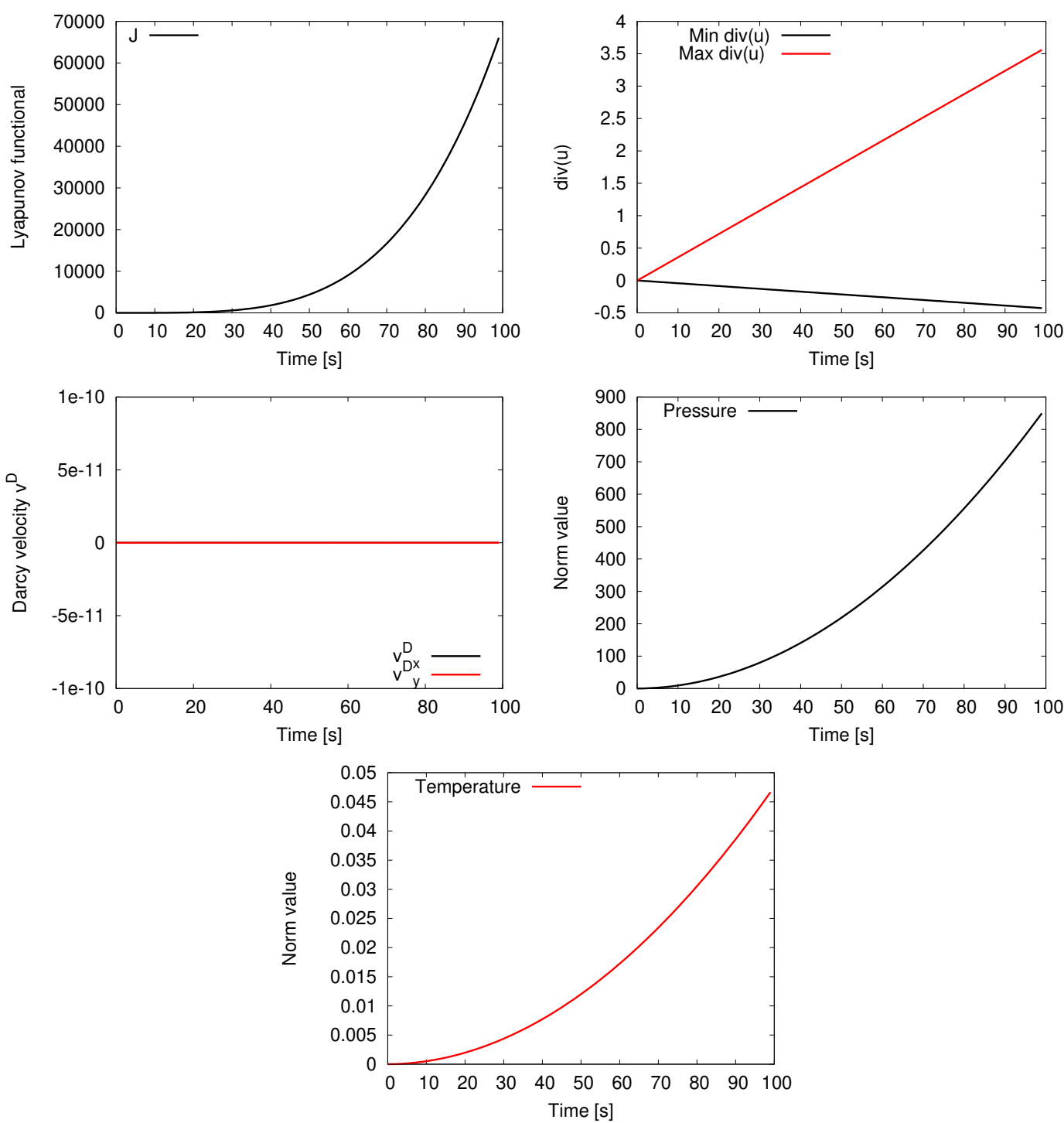

Figure 9: Example 3 (pressure source). Evolution of the Lyapunov functional $J$, divu, the components of $\mathbf{v}^{D}$ and the norms of the pressure and temperature, i.e., $\|p\|_{L^{2}}$ and $\|T\|_{L^{2}}$, respectively.

\subsection{Example 4: A heat source $H^{j}$}

In this example, we keep all material parameters, boundary conditions and initial data as before and take now a nonzero heat source, while setting $F^{j}=Q^{j}=0$. To this end, we use:

$$
H^{j}=t * \exp \left(-f\left(\left(x-x_{c}\right)^{2}+\left(y-y_{c}\right)^{2}\right)\right), \quad x_{c}=y_{c}=0.5,
$$


with $f=100$ and a parameter $t=t_{j}, j=0, \ldots, N$ with $N=100$.
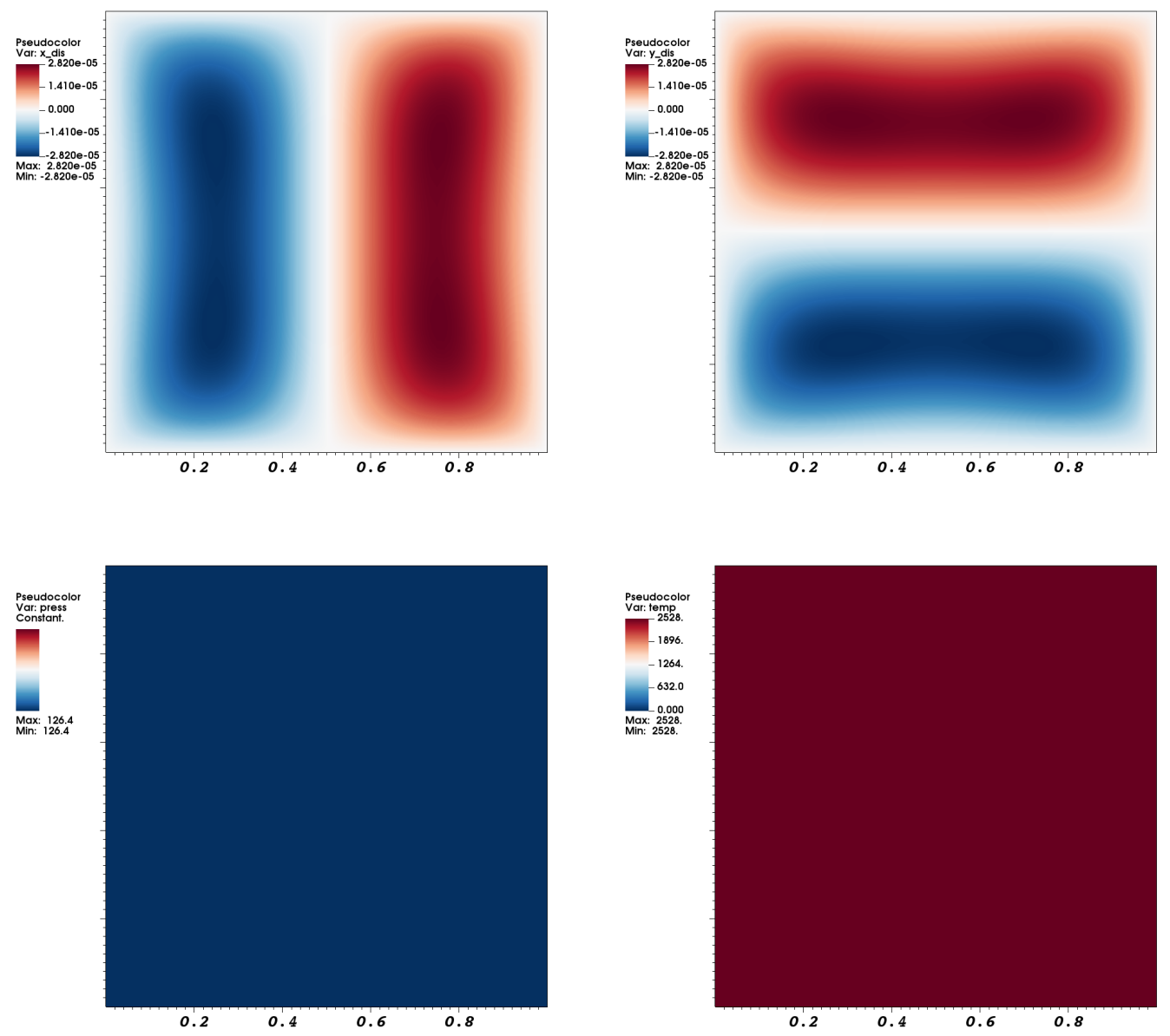

Figure 10: Example 4 (heat source). Going from top left to bottom right: $\mathbf{u}_{x}, \mathbf{u}_{y}, p, \vartheta$ at $t=100 \mathrm{~s}$ with $N=100$. The pressure and temperature fields are nearly constant, which can be justified by carefully studying the underlying system and also taking the boundary conditions into account. 

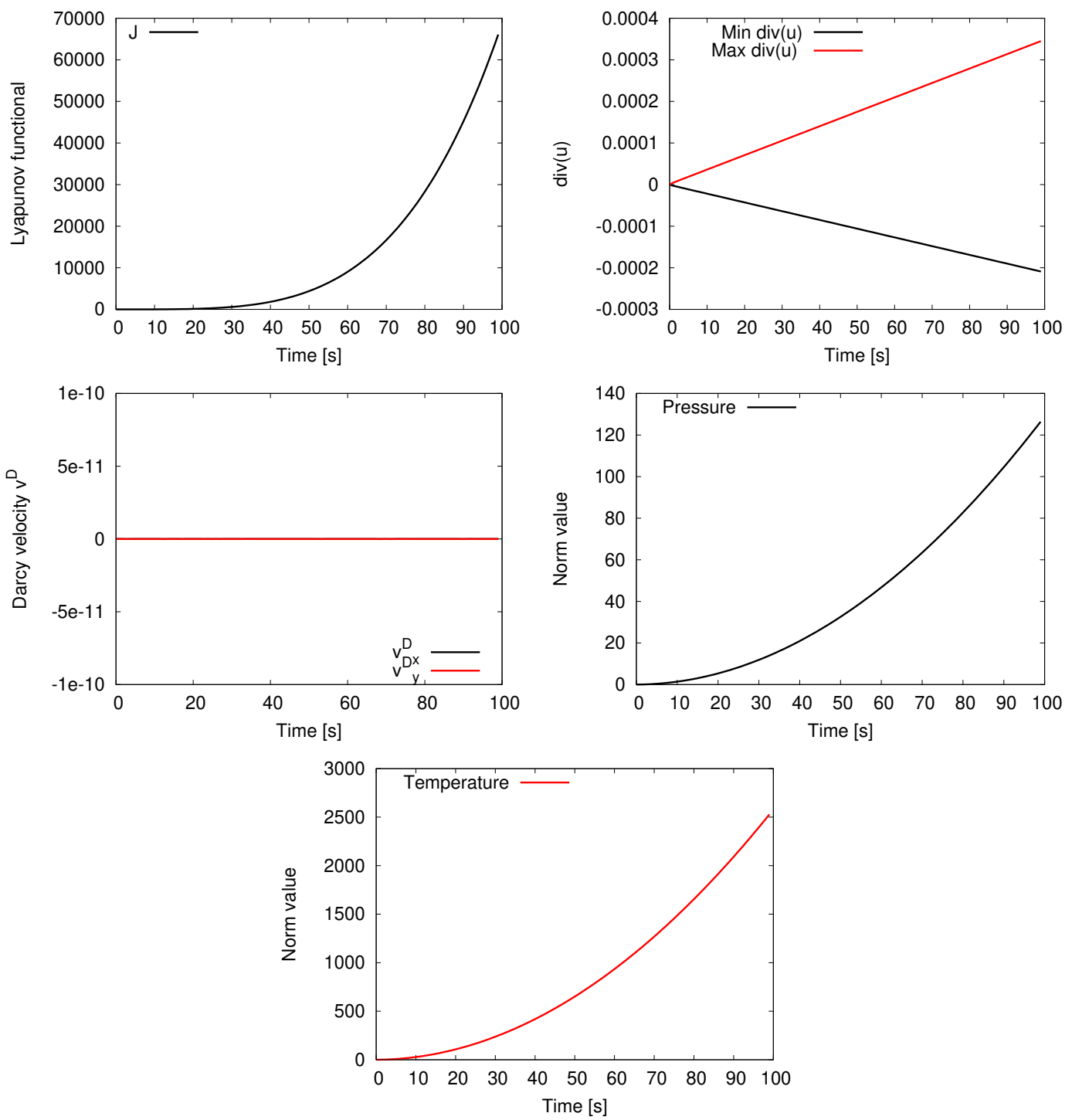

Figure 11: Example 4 (heat source). Evolution of the Lyapunov functional $J$, divu, the components of $\mathbf{v}^{D}$ and the norms of the pressure and temperature, i.e., $\|p\|_{L^{2}}$ and $\|T\|_{L^{2}}$, respectively.

\section{Conclusion}

In this paper we have undertaken a study of the equations of semi-linear thermoporoelasticity in their incremental- i.e. time discrete - form. We prove existence of a weak solution and use the natural free energy to construct a Lyapunov functional. This implies global stability of the solution in the sense that one can repeat the stepping procedure an arbitrary number of times. 
Our results apply to the setting where the fluid velocity is more important than the velocity of the structure. In the vocabulary of Coussy [5] it is an Eulerian formulation, where the main part of the velocity is the Darcy velocity, i.e. the relative velocity between fluid and skeleton. The characteristic time of interest for us is the heat convection time. We suppose a growth condition on the dimensionless heat diffusion coefficient $\beta(\vartheta)$ in order to control the convection term for moderate Péclet number.

It is well-known that the heat diffusion coefficient is a strictly increasing function of temperature. In the range of the temperature variations considered in this paper, it is practically constant. The data, together with a discussion, are presented in Remark 1 , and the conclusion is that the nonlinear regularization of the heat diffusion coefficient by the term $C_{m i n} \vartheta^{2}$ is negligible for the physical regime of the temperatures.

The nonlinearity of $\beta$ plays a role only for large values of $\vartheta$, were the validity of the model is not clear any more.

Finally, in our numerical tests, we proposed characteristic settings in order to study the behavior of the different solution components. Using a manufactured solution, we could show very good agreements in Example 1 between analytical suggestions and the numerically-computed values. Moreover in Example 2, we could computationally show the robustness with respect to mesh refinement. Moreover, our findings were studied in terms of several quantities of interest. The behavior of the Lyapunov functional, the volume strain and the Darcy pressures are all plausible and confirm with our theoretical findings.

\section{References}

\section{References}

[1] D. Arndt and W. Bangerth and T. C. Clevenger and D. Davydov and M. Fehling and D. Garcia-Sanchez and G. Harper and T. Heister and L. Heltai and M. Kronbichler and R. M. Kynch and M. Maier and J.-P. Pelteret and B. Turcksin and D. Wells, The deal. II Library, Version 9.1, J. Numer. Math., accepted for publication, 2019, 10.1515/jnma-2019-0064.

[2] Bedford A., Drumheller D.S. : A variational theory of immiscible mixtures. Arch. Ration. Mech. Anal. 68 (1978), 37-51.

[3] M.A. Biot, Mechanics of Deformation and Acoustic Propagation in Porous Media, J. Appl. Phys. 33 (1962), 1482.

[4] P. G. Ciarlet, The finite element method for elliptic problems, North-Holland, 1987. 
[5] O. Coussy, Poromechanics, John Wiley and Sons, 2004.

[6] O. Coussy, L. Dormieux, E. Detournay, From mixture theory to Biot's approach for porous media, Int. J. Solids Struct. 35(34) (1998), 4619-4635.

[7] C.J. van Duijn, A. Mikelić, M. F. Wheeler, T. Wick, Thermoporoelasticity via homogenization I. Modeling and formal two-scale expansions, Internat. J Engng Sci., 138 (2019), 1-25.

[8] C.J. van Duijn, A. Mikelić, Mathematical Theory of Nonlinear Single-Phase Poroelasticity, preprint of the Darcy Center Eindhoven-Utrecht, The Netherlands, June 2019, www.darcycenter.org/wp-content/uploads/2019/06/Duijnand-Mikelic-2019-5.pdf

[9] C. K. Lee, C. C. Mei, Thermal consolidation in porous media by homogenization theory-I. Derivation of macroscale equations, Adv. Water Res. 20 (1997), 127144 .

[10] C. K. Lee, C. C. Mei, Thermal consolidation in porous media by homogenization theory-II. Calculation of effective coefficients, Adv. Water Res. 20 (1997), 145156.

[11] R. W. Lewis, B. A. Schrefler, The Finite Element Method in the Static and Dynamic Deformation and Consolidation of Porous Media, John Wiley and Sons, 1998.

[12] J.L. Lions, Quelques méthodes de résolution des problémes aux limites non linéaires. Dunod, Paris, (1969).

[13] D. F. McTigue, Thermoelastic Response of Fluid-Saturated Porous Rock, J. Geophys. Res. 91 (1986), 9533-9542.

[14] C.C. Mei, B. Vernescu, Homogenization methods for multiscale mechanics. World scientific, (2010).

[15] A. Mikelić, M.F. Wheeler, On the interface law between a deformable porous medium containing a viscous fluid and an elastic body. Math. Models Methods Appl. Sci. 22 (2012), 1250031.

[16] D. Néron, D. Dureisseix. A computational strategy for thermo-poroelastic structures with a time-space interface coupling. Int. J. for Numer. Meth. Eng. 75 (2007), 1053-1084. 
[17] N. Noii and T. Wick: A phase-field description for pressurized and nonisothermal propagating fractures, Comput. Method. Appl. M., 351 (2019), 860890.

[18] T. Roubiček, Nonlinear Partial Differential Equations with Applications. Springer Science \& Business Media, (2005).

[19] J. Rutqvist, L. Börgesson, M. Chijimatsu, A. Kobayashi, L. Jing, T.S. Nguyen, J. Noorishad, C.F. Tsang, Thermohydromechanics of partially saturated geological media: governing equations and formulation of four finite element models. Int. J. Rock Mech. Min. Sci. 38, 105-127 2001.

[20] E. Sanchez-Palencia E., Non-Homogeneous Media and Vibration Theory, Springer Lecture Notes in Physics 129, Springer, (1980).

[21] D. Tran, A. T. Settari and L. Nghiem, Predicting growth and decay of hydraulicfracture witdh in porous media subjected to isothermal and nonisothermal flow. SPE J., 18 (2013), 781-794.

[22] T. Wick, Solving monolithic fluid-structure interaction problems in arbitrary Lagrangian Eulerian coordinates with the deal. II library. Arch. Numer. Software, Vol. 1 (2013), pp. 1-19. 